\title{
Polydispersed flow modelling using population balances in an adaptive mesh finite element framework
}

\author{
Gaurav Bhutani ${ }^{\mathrm{a}, \mathrm{b}, *}$, Pablo R. Brito-Parada ${ }^{\mathrm{a}, \mathrm{b}}$, Jan J. Cilliers $^{\mathrm{a}}$ \\ ${ }^{a}$ Rio Tinto Centre for Advanced Mineral Recovery, Department of Earth Science and Engineering, Imperial College London, South Kensington Campus, London \\ SW7 2AZ, United Kingdom \\ ${ }^{b}$ Applied Modelling and Computation Group, Department of Earth Science and Engineering, Imperial College London, South Kensington Campus, London SW7 \\ $2 A Z$, United Kingdom
}

\begin{abstract}
An open-source finite element framework to model multiphase polydispersed flows is presented in this work. The Eulerian-Eulerian method was coupled to a population balance equation and solved using a highly-parallelised finite element code-Fluidity. The population balance equation was solved using DQMOM. A hybrid finite element-control volume method for solving the coupled system of equations was established. To enhance the efficiency of this solver, fully-unstructured non-homogeneous anisotropic mesh adaptivity was applied to systematically adapt the mesh based on the underlying physics of the problem. This is the first time mesh adaptivity has been applied to the external coordinates of the population balance equation for modelling polydispersed flows. Rigorous model verification and benchmarking were also performed to demonstrate the accuracy of this implementation. This finite element framework provides an efficient alternative to model polydispersed flow problems over the other available finite volume CFD packages.
\end{abstract}

Keywords: Polydispersed flow, Population balance modeling, DQMOM, Multiphase flow, Finite element method, Mesh adaptivity

\section{Introduction}

Multiphase polydispersed flows are essential elements of the chemical process industry and are found extensively in pharmaceutical, minerals processing and food processing operations, to name a few. Some of the applications where polydispersity plays a prominent role are fluidised beds, chemical reactors, bubble columns, and the spray of fuels in automotive and aircraft engines (Marchisio and Fox, 2013). A good understanding and prediction of these flows is therefore crucial for the optimum design of chemical process systems.

The term polydispersity refers to the heterogeneity of the dispersed particles, which may exist due to the variation in particle size or any other particle property in space. Developing and performing experiments for the applications involving multiphase polydispersed flows is extremely challenging and becomes expensive when one is interested in exploring different operating conditions and designs. Computational Fluid Dynamics (CFD) on the other hand provides a cost effective tool to carry out the aforementioned tasks and study scale-up. Until a few decades ago it was extremely challenging to model complex industrial flow systems, but with the advent of parallelised machines and improved algorithms it has become possible to approach these problems computationally.

\footnotetext{
${ }^{*}$ Corresponding author

Email addresses: g.bhutani12@imperial.ac.uk (Gaurav Bhutani), pbritopa@imperial.ac.uk (Pablo R. Brito-Parada),

j.j.cilliers@imperial.ac.uk (Jan J. Cilliers)
}

Even though the computational power and techniques have advanced tremendously over the past few years, chemical engineers interested in modelling polydispersed flows are still constrained by the available (commercial and non-commercial) CFD software products. There are not many options available when it comes to modelling polydispersed flows and the ones available do not utilise the mathematical resources efficiently, and hence are sub-optimal. This paper introduces a computationally-efficient, open-source, Finite Element (FE) framework to model polydispersed flows using parallelised adaptive mesh refinement.

Dispersed particles are commonly modelled using two different approaches - the Lagrangian way and the Eulerian way. The continuous phase, on the other hand, is treated as a continuum in most approaches and is therefore modelled using the Eulerian method. This leads to the popular Eulerian-Lagrangian and the Eulerian-Eulerian methods for simulating multiphase flows (Prosperetti and Tryggvason, 2007). The Eulerian-Lagrangian method can become prohibitively expensive when the number of dispersed particles is large and due to this reason the literature on polydispersed flow modelling in chemical engineering research has been dominated by the Eulerian-Eulerian method in the past few years.

Multiphase Eulerian-Eulerian flow equations coupled to a Population Balance Equation (PBE) have been used by many to model polydispersed flow systems in the last decade (Chen et al., 2004; Bannari et al., 2008; Buffo et al., 2012). PBE, 
which is a conservation equation for the dispersed particles, is used to model the evolution of the particle size distribution (Ramkrishna, 2000). Although different methods have been proposed to solve the PBE, one method that has attracted special attention from the research community is the method of moments (Frenklach and Harris, 1987), particularly for polydispersed flow modelling where the PBE is coupled to the Eulerian-Eulerian multiphase flow equations. Method of moments encapsulates a general family of methods depending the type of closure used to close the approximated moment set. Two of the popular methods of this type are the Quadrature Method of Moments (QMOM) (McGraw, 1997) and the Direct Quadrature Method of Moments (DQMOM) (Marchisio and Fox, 2005).

Zucca et al. (2006) successfully demonstrated the coupling of DQMOM to the flow equations in solving a polydispersed flow problem. DQMOM provides an efficient closure and is more economical when compared to the other methods to solve PBEs. Marchisio and Fox (2005) showed that the PBE can be approximated to a good accuracy by as few as four moment equations in DQMOM. This is a very small number of equations when compared to the method of classes - the other method used to solve PBEs (Marchal et al., 1988; Sanyal et al., 2005; Bannari et al., 2008). This particular advantage over other PBE solution methods, along with its adaptive quadrature approach, has inspired many researchers to use DQMOM to model polydispersed flows lately (Zucca et al., 2006; Selma et al., 2010; Chan et al., 2010).

However, most implementations of the models to solve polydispersed flows have been in the following software packages:

1. OpenFOAM ${ }^{\circledR}$ (an open-source finite volume software): Silva and Lage (2011); Selma et al. (2010); Becker et al. (2014); Favero et al. (2015).

2. ANSYS $^{\circledR}{ }^{\circledR}$ Fluent ${ }^{\circledR}$ (a commercial finite volume software): Zucca et al. (2006); Buffo et al. (2012); Szilágyi et al. (2014).

3. $\mathrm{ANSYS}^{\circledR} \mathrm{CFX}^{\circledR}$ (a commercial control volume finite element software): Silva et al. (2008); Yeoh et al. (2012).

Engineers, in some sense, have been limited by the use of these solvers when modelling polydispersed flows and there has not been much discussion in the literature to improve their efficiency. In fact, having an efficient solver is as important as having a good model because a non-optimal solver can sometimes force the engineer to revert to a crude model to make it tractable for solving industrial design problems. There have been some efforts recently to use novel computational techniques for solving the population balance equation (Prakash et al., 2013; Santos et al., 2013), yet there does not exist an efficient framework that can handle an industrial-scale heterogeneous problem. In order to address this issue, DQMOM was implemented in a finite element framework in this work and was coupled to the Eulerian-Eulerian method to solve multiphase polydispersed flow problems. All equations were solved using the open-source multiphase CFD code-Fluidity, which solves the Navier-Stokes and accompanying field equations on unstructured meshes using the finite element method (AMCG, 2015). This framework not only provides a new open-source alternative to the available software packages for solving polydispersed flow equations, but it also provides the features of the finite element method-like higher order discontinuous Galerkin and continuous Galerkin methods-compared to the other software packages employing the finite volume/ control volume methods.

Most CFD packages solve the flow equations on fixed meshes that do not change as the simulation progresses. These meshes may allow for anisotropy and/or inhomogeneity based on the initial condition or a general understanding of the flow physics (e.g. boundary layer flow) but are rarely optimum for a transient CFD problem. In reality, almost every transient simulation involves one or more time evolving phenomena, which drive the varying spatial requirement for the optimal placement of the mesh nodes. Therefore, to enhance the efficiency of the solution method in this work, fully unstructured, nonhomogeneous, anisotropic mesh adaptivity was applied in a parallelised environment to systematically adapt the mesh based on the underlying physics of the problem (Piggott et al., 2009). Improved solution efficiency through optimised adaptive meshes will make it possible to compute large-scale flows in a tractable manner. To the best of the authors' knowledge, this is the first time adaptive mesh refinement has been applied to the external coordinates of the population balance equation for modelling polydispersed flows.

The remaining part of this paper is organised as follows. Section 2 describes the model that was used to solve the polydispersed flow problem. It starts with a discussion on the formulation of the flow equations and the population balance equation, followed by a brief explanation of the finite element implementation of the coupled system and the solution algorithm. The section closes with a discussion of the mesh adaptivity technique that was utilised to improve the efficiency of the solution method in this paper. Section 3 shows model verification results for the DQMOM implemention described in this work. The polydispersed flow problem that was solved and the corresponding results are presented in Section 4; results for a monodispersed case are presented first, followed by a fully polydispersed flow problem. The improvements achieved by the use of mesh adaptivity are also quantified and discussed in this section. Finally, the conclusions of this work are summarised in Section 5.

\section{Model description and solution}

\subsection{Flow equations}

In general, polydispersed flows consist of one continuous phase and one (or more) dispersed phase(s). For simplicity of discussion, a problem consisting of one dispersed and one continuous phase was solved on a 2D domain in this work, although all 
methods were implemented to be able to handle multiple dispersed phases in up to three spatial dimensions.

Interpenetrating multiphase flows are generally modelled using three different approaches depending on the flow regime, problem complexity and the availability of computational resources. The simplest technique is the mixture model where only one momentum equation is solved for an average mixture phase (Bowen, 1976). All other phase relative velocities are computed from an empirical or an analytical equation. For this reason, the mixture model is not able to predict the dispersed phase velocity field accurately. A better but more complex model is the Eulerian-Eulerian multiphase model, where a separate momentum equation for each phase is solved. All phases in this method are assumed to be continuous and a model for the interphase force terms, which couple the momentum equations between any two phases, is needed. The third, and the most expensive, model is the Eulerian-Lagrangian model. As the name suggests, all dispersed particles are modelled as discrete objects with an Eulerian flow model for the continuous phase. This approach can become prohibitively expensive when the number of particles is large.

Considering the limitations of the mixture model and the Eulerian-Lagrangian model, an incompressible multiphase Eulerian-Eulerian method was chosen to model the two phases in this work. This method offered a practical approach to model polydispersed flows over the other aforementioned techniques.

In the Eulerian-Eulerian method, a volume-averaged momentum equation is derived for each phase over volumes of appropriate sizes (Brennen, 2005). These representative volumes should be large enough to contain several dispersed particles and small enough for the gradients of the volume-averaged flow properties to remain continuous. This volume averaging gives rise to a conserved scalar called volume fraction and the momentum equation for phase $i$, in terms of this volume fraction, is given as (Brennen, 2005):

$$
\alpha_{i} \rho_{i} \frac{\partial \mathbf{u}_{i}}{\partial t}+\alpha_{i} \rho_{i} \mathbf{u}_{i} \cdot \nabla \mathbf{u}_{i}=-\alpha_{i} \nabla p+\alpha_{i} \rho_{i} \mathbf{g}+\nabla \cdot\left(\alpha_{i} \overline{\bar{\tau}}_{i}\right)+\mathbf{f}_{i},
$$

where the phase $i$ can be continuous or dispersed, given in this text by $c$ and $d$ respectively. $\alpha_{i}$ is the volume fraction of phase $i, \rho_{i}$ is the phase density, $\mathbf{u}_{i}$ is the phase velocity, $p$ is the static pressure, $\mathbf{g}$ is the acceleration due to gravity, $\overline{\bar{\tau}}_{i}$ is the deviatoric stress tensor, and $\mathbf{f}_{i}$ is the interphase force term. Note that Einstein's notation is not used in Equation (1).

The phases in Equation (1) were modelled as interpenetrating continua with each phase having its own velocity field so that mixing could be modelled effectively. There was a common pressure field considered for all the phases, as can be seen from Equation (1). This approximation is reasonable in situations where the relative velocity between the phases is small and the change in dispersed phase volume is negligible (Yeoh et al., 2013). Separate pressure fields for the two phases may be needed in the case of the above conditions not being satisfied. The common pressure field approximation simplified the flow equations as only one continuity equation had to be satisfied for all phases. The combined continuity equation that was solved in this work is given by (Brennen, 2005):

$$
\sum_{i=1}^{N_{\text {phases }}} \nabla \cdot\left(\alpha_{i} \mathbf{u}_{i}\right)=0 .
$$

The viscous stress term in Equation (1) was modelled as

$$
\nabla \cdot\left(\alpha_{i} \overline{\bar{\tau}}_{i}\right)=\nabla \cdot\left(\alpha_{i} \mu_{i} \nabla \mathbf{u}_{i}\right)
$$

as described in Jacobs et al. (2013), where $\mu_{i}$ is the dynamic viscosity of phase $i$. The momentum equations for the two phases and the combined continuity equation, Equations (1) and (2) respectively, were solved in conjunction with the following two equations for the volume fractions, to get the complete set of flow variables:

$$
\frac{\partial \alpha_{d}}{\partial t}+\nabla \cdot\left(\alpha_{d} \mathbf{u}_{d}\right)=0
$$

which was solved to get the dispersed phase volume fraction $\alpha_{d}$ and the conservation equation

$$
\sum_{i=1}^{N_{\text {phases }}} \alpha_{i}=1
$$

which was used to obtain continuous phase volume fraction $\alpha_{c}$. No turbulence model was solved in this work as all flow conditions were laminar.

In polydispersed flow modelling, the most important term in the momentum equation is the interphase force $\mathbf{f}_{i}$. This includes forces such as drag, lift and the virtual mass force acting on phase $i$ from all other phases. In the present work, only the contribution from the drag force was considered for the interphase force term and the other two forces were neglected, same as Chen et al. (2004). The interphase drag force can be written as:

$$
f_{D}=\frac{3 \alpha_{c} \alpha_{d} C_{D} \rho_{c}\left(\mathbf{u}_{c}-\mathbf{u}_{d}\right)\left|\mathbf{u}_{c}-\mathbf{u}_{d}\right|}{4 d}
$$

where $C_{D}$ is the drag coefficient and $d$ is the dispesed phase diameter. The popularly used Schiller-Naumann drag coefficient (Schiller and Naumann, 1935) was selected for this work. It is given as:

$$
C_{D}= \begin{cases}\frac{24}{R e_{d}}\left(1+0.15 R e_{d}^{0.687}\right) & \text { if } R e_{d}<1000, \\ 0.44 & \text { otherwise, }\end{cases}
$$

where $R e_{d}$, the dispersed phase (or particle) Reynolds number, is defined as:

$$
R e_{d}=\frac{\rho_{c} d\left|\mathbf{u}_{c}-\mathbf{u}_{d}\right|}{\mu_{c}} .
$$

The interphase drag force can have a strong dependence on the dispersed phase diameter $d$ (Tabib et al., 2008), and choosing a correct value for $d$ becomes essential when solving a polydispersed flow system. In many works, the value of $d$, obtained 
from experiments, has been considered constant over the whole domain for simplicity (McClure et al., 2014). Polydispersed flows on the other hand, by definition, have a dispersed phase whose size varies over space and time, and this variation can be significant in many cases. For this reason, a population balance equation was solved to accurately model the polydispersity of the flow, which is discussed next.

\subsection{Population balance equation}

The Population Balance Equation (PBE) is simply a conservation equation for the number of dispersed particles, which is used to model the evolution of the particle size distribution (Ramkrishna, 2000). As discussed previously, the need for a population balance equation arises for the prediction of particle size distribution to accurately model the drag force term in momentum equations (Equation (1)). Additionally, it may also be important to have some knowledge of the distribution of particle sizes in the domain for a general understanding of the flow phenomenon. It must be noted that 'particle size' and 'dispersed phase size' have been, and will be, used interchangeably and they mean the same thing in the context of this work.

The particle size distribution is characterised by a Number Density Function (NDF) that is a function of variables in the particle state space, which consists of external and internal coordinates. External coordinates are the spatial coordinates whereas internal coordinates may include particle specific properties like particle size, age, moisture content, etc.

The generalised population balance equation is an integrodifferential equation that can model flow of particles, diffusion in the external and internal space, particle growth, and the coalescence and breakage of particles. The PBE applied in this work was given by:

$$
\frac{\partial n(\xi ; \mathbf{x}, t)}{\partial t}+\nabla \cdot(\langle\mathbf{u} \mid \xi\rangle n)=S_{\xi}(\xi ; \mathbf{x}, t)
$$

where $n(\xi ; \mathbf{x}, t)$ is the dispersed phase number density function, with $\mathbf{x}$ and $\xi$ being the external and the internal coordinates respectively. There was only one internal coordinate considered in this work and that coordinate represented the dispersed phase size.

In Equation (9), $\langle\mathbf{u} \mid \xi\rangle$ is the dispersed phase velocity field conditional to the particle size $\xi$, and $S_{\xi}$ includes all the source terms for the PBE. $S_{\xi}$ in this work included the birth and death functions due to particle breakage and coalescence and was given by:

$$
S_{\xi}=B_{B}+B_{C}-D_{B}-D_{C}
$$

The birth and death functions due to breakage were given as:

$$
B_{B}=\int_{\xi}^{\infty} v\left(\xi_{1}\right) a\left(\xi_{1}\right) b\left(\xi \mid \xi_{1}\right) n\left(\xi_{1}\right) \mathrm{d} \xi_{1}
$$

and

$$
D_{B}=a(\xi) n(\xi),
$$

respectively. Here, $v(\xi), a(\xi)$ and $b\left(\xi \mid \xi_{1}\right)$ are the breakage kernels that define the number of particles formed after breakage, the breakage frequency, and the daughter distribution function, respectively.

Correspondingly, the birth and death functions due to coalescence were given as:

$$
B_{C}=\frac{1}{2} \int_{0}^{\xi}\left(\frac{\xi^{2}}{\xi^{\prime 2}}\right) \beta\left(\xi^{\prime}, \xi_{1}\right) n\left(\xi^{\prime}\right) n\left(\xi_{1}\right) \mathrm{d} \xi_{1}
$$

and

$$
D_{C}=\int_{0}^{\infty} \beta\left(\xi, \xi_{1}\right) n(\xi) n\left(\xi_{1}\right) \mathrm{d} \xi_{1},
$$

respectively, where $\beta\left(\xi^{\prime}, \xi_{1}\right)$ is the coalescence frequency for particles of sizes $\xi^{\prime}$ and $\xi_{1}$. In Equation (13), $\xi^{\prime}$ is given as $\xi^{3}=\xi^{3}-\xi_{1}^{3}$, i.e. the particles are considered three-dimensional and the volume of the resulting particle class $(\xi)$ is the sum of the volumes from the contributing size classes $\left(\xi^{\prime}\right.$ and $\left.\xi_{1}\right)$. A detailed derivation of these terms can be found in Ramkrishna (2000).

The presence of an internal coordinate in the number density function, $n(\xi ; \mathbf{x}, t)$, makes it difficult to solve the integrodifferential PBE (Equation (9)). For this reason, the method of classes was developed by Marchal et al. (1988), which discretises the internal coordinate into various classes. Each class gives rise to an advection-diffusion equation for the NDF corresponding to that class. For a reasonable solution accuracy, a large number of classes need to be considered, which requires solving many coupled advection-diffusion equations to predict the particle size distribution. Since the PBE is coupled with multiphase flow equations, this makes the solution of a normal engineering system extremely expensive.

To circumvent this problem, the method of moments becomes useful. As the name suggests, in this method, instead of solving for the NDF, equations for the moments of the NDF are solved. In multiphase flow modelling, where only the dispersed phase diameter $d$ is needed as an input from the population balance equation, as few as four moments can be enough in most cases (Marchisio and Fox, 2005). The $k^{\text {th }}$ moment of the number density function is defined as:

$$
m_{k}=\int_{0}^{\infty} \xi^{k} n(\xi) \mathrm{d} \xi
$$

The well-known Sauter Mean Diameter (SMD) defined in terms of the moments as

$$
d_{32}=\frac{m_{3}}{m_{2}}
$$

was used as the representative diameter in the interphase drag force relation (Equation (6)).

Various methods of moments exist in the literature, which fundamentally differ in the way they close the set of moment equations. The Direct Quadrature Method of Moments (DQMOM) developed by Marchisio and Fox (2005) was used in this work. 
This method uses a quadrature approximation to the NDF, given by:

$$
n(\xi ; \mathbf{x}, t)=\sum_{j=1}^{N} w_{j}(\mathbf{x}, t) \delta\left[\xi-\langle\xi\rangle_{j}(\mathbf{x}, t)\right],
$$

as opposed to approximating the integrals in other methods of moments. $\delta$ in the above equation is the Dirac delta function, $N$ is the total number of quadrature points, and $w_{j}$ and $\langle\xi\rangle_{j}$ are the weights and abscissas respectively in the DQMOM approximation.

Taking moments of Equation (9) after substituting Equation (17) for the NDF gives the DQMOM form of the population balance equation:

$$
\frac{\partial w_{j}}{\partial t}+\nabla \cdot\left(\langle\mathbf{u}\rangle_{j} w_{j}\right)=g_{j}
$$

and

$$
\frac{\partial \varsigma_{j}}{\partial t}+\nabla \cdot\left(\langle\mathbf{u}\rangle_{j} \varsigma_{j}\right)=h_{j}
$$

where $j=1,2, \ldots, N$. The source terms $g_{j}$ and $h_{j}$ were obtained from the solution of the linear system

$$
(1-k) \sum_{j=1}^{N}\langle\xi\rangle_{j}^{k} g_{j}+k \sum_{j=1}^{N}\langle\xi\rangle_{j}^{k-1} h_{j}=\bar{S}_{k}^{(N)},
$$

where $k=1,2, \ldots, 2 N$. Although the advection terms in Equations (18) and (19) included a velocity field conditional on the abscissa $\langle\xi\rangle_{j}$, a common velocity field approximation to the dispersed phase was used in this work without any dependence on the particle size. $\bar{S}_{k}^{(N)}$ in Equation (20) is the $k^{\text {th }}$ moment of the source term of the PBE with the DQMOM quadrature approximation applied. A detailed formulation of the breakage and aggregation integrals, which constitute the source term $\bar{S}_{k}^{(N)}$, can be found in Marchisio and Fox (2005) for the DQMOM framework.

Equations (18) and (19) form a system of combined advection equations that are coupled in the source terms. These equations were solved for the weights $w_{j}$ and the weighted abscissas $\varsigma_{j}$, which were then used to calculate the moments and eventually the Sauter mean diameter $d_{32}$. The major advantages of using DQMOM over any other method of moments is that each weight and abscissa can be defined as a function of space, which makes it easier to implement this method, and very few abscissas are needed to accurately model the NDF due to the adaptive quadrature approach. Additionally, each node can have its own velocity field (if required) as stated in Marchisio and Fox (2005). More details on the DQMOM derivation of the population balance equation can be seen in the work by Marchisio and Fox (2005).

\subsection{Finite element implementation}

Sections 2.1 and 2.2 presented the equations that were chosen to model the polydispersed system in this work. This section describes the Finite Element (FE) discretisation and the solution method that were implemented in Fluidity's framework to solve the polydispersed flow equations. Fluidity is an open-source, multiphase CFD software that can be used to solve flow and accompanying field equations using the Finite Element Method (FEM) on unstructured meshes (AMCG, 2015). DQMOM was implemented in Fluidity, as a part of this work, to solve the population balance equation and was integrated to the multiphase flow solver subroutines to model polydispersed flows.

\subsubsection{Numerical discretisation}

For illustration, the FE discretisation process is demonstrated for an advection-diffusion equation. Using the Galerkin finite element method, the weak form of the advection-diffusion equation was written by multiplying it with a test function $\widetilde{w}$ and integrating over a region $\Omega$,

$$
\int_{\Omega} \widetilde{w}\left(\frac{\partial c}{\partial t}+\nabla \cdot(\mathbf{u} c)-\nabla \cdot(\overline{\bar{K}} \cdot \nabla c)\right) \mathrm{d} \Omega=\int_{\Omega} \widetilde{w} S \mathrm{~d} \Omega .
$$

$c$ in the above equation is the unknown scalar, and $\overline{\bar{\kappa}}$ and $S$ are the diffusivity tensor and the source term, respectively. Both $\widetilde{w}$ and $c$ in the Galerkin method come from the same space- the Sobolev space (Elman et al., 2014), in this case.

Equation (21) was discretised in Fluidity by taking a finitedimensional approximation of the unknown $c$ as $c=\sum_{i=1}^{N_{\text {nodes }}} c_{i} \phi_{i}$. In this approximation, $\phi_{i}$ are the basis functions for this finitedimensional subspace that took the value one at the node $i$ and zero at all other nodes. These basis functions in Fluidity were piecewise polynomial functions, which could be continuous or discontinuous at the element boundary (AMCG, 2015).

The final discrete equation that was solved in Fluidity can be written in the matrix form as:

$$
\mathrm{M} \frac{d \underline{c}}{d t}+\mathrm{A}(\mathbf{u}) \underline{c}+\mathrm{K} \underline{c}=\mathrm{M} \underline{S}
$$

where $\underline{c}$ is the vector of coefficients representing the unknown scalar $\bar{c}$, and M, A and $\mathrm{K}$ are the mass, advection and diffusion matrices, respectively. This matrix equation was solved using an iterative solver to get the finite-dimensional approximation of the unknown $c$.

The Galerkin finite element method discussed above was used to solve the momentum equations in this work using a pressure projection approach, as explained by Jacobs et al. (2013). On the other hand, a node-centred Control Volume (CV) method was used to discretise the population balance equation to ensure conservation (Wilson, 2009). The volume fraction equation was also discretised using the CV method. Hence, a hybrid Finite Element-Control Volume (FE-CV) method was established to solve the polydispersed flow equations in this work.

In a typical case, the velocity, pressure and population balance fields were discretised using a $\mathrm{P} 1_{\mathrm{DG}}$, a $\mathrm{P} 2_{\mathrm{CG}}$ and a $\mathrm{P} 0_{\mathrm{CV}}$ method, respectively. P0, P1 and P2 refer to the use of piecewise constant, piecewise linear and piecewise quadratic polynomial shape functions, respectively. These shape functions can 
(a)
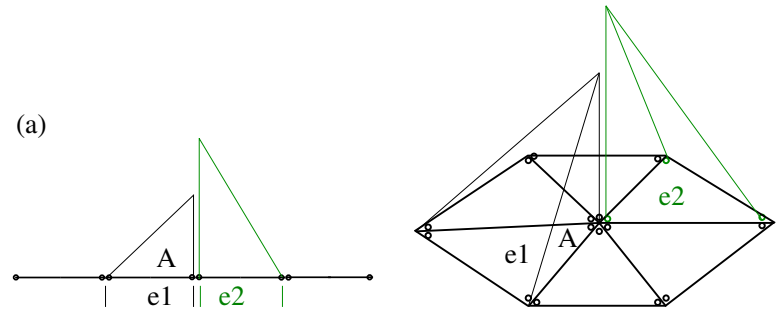

(b)
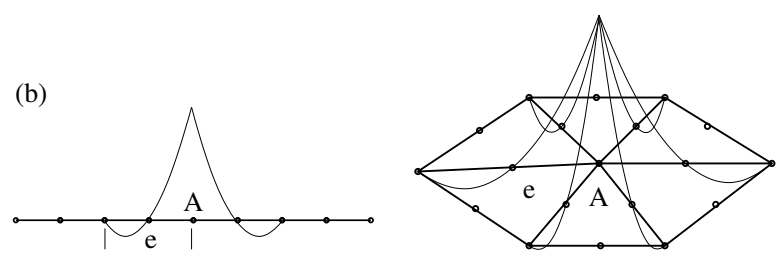

(c)
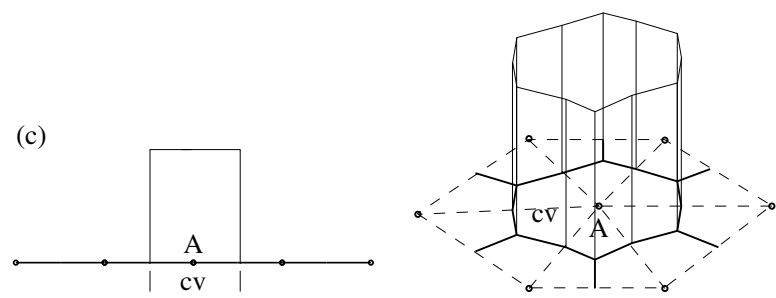

Figure 1: Diagram illustrating the elements and the corresponding shape functions in the different methods used to discretise velocity, pressure and population balance fields on $1 \mathrm{D}$ (left) and 2D (right) FE meshes. (a) P1 DG method: linear (P1) shape functions are discontinuous across the vertex A separating the two elements e1 and e2. Each element has its its own node corresponding to vertex A. (b) $\mathrm{P} 2_{\mathrm{CG}}$ method: quadratic (P2) shape functions are continuous across the vertex A. Each element shares the same node corresponding to vertex $\mathrm{A}$. (c) $\mathrm{P}_{\mathrm{CV}}$ method: constant $(\mathrm{P} 0)$ shape functions are defined over the control volume, which is formed by joining the centroids of the elements sharing the vertex A. Figure taken from the Fluidity Manual (AMCG, 2015).

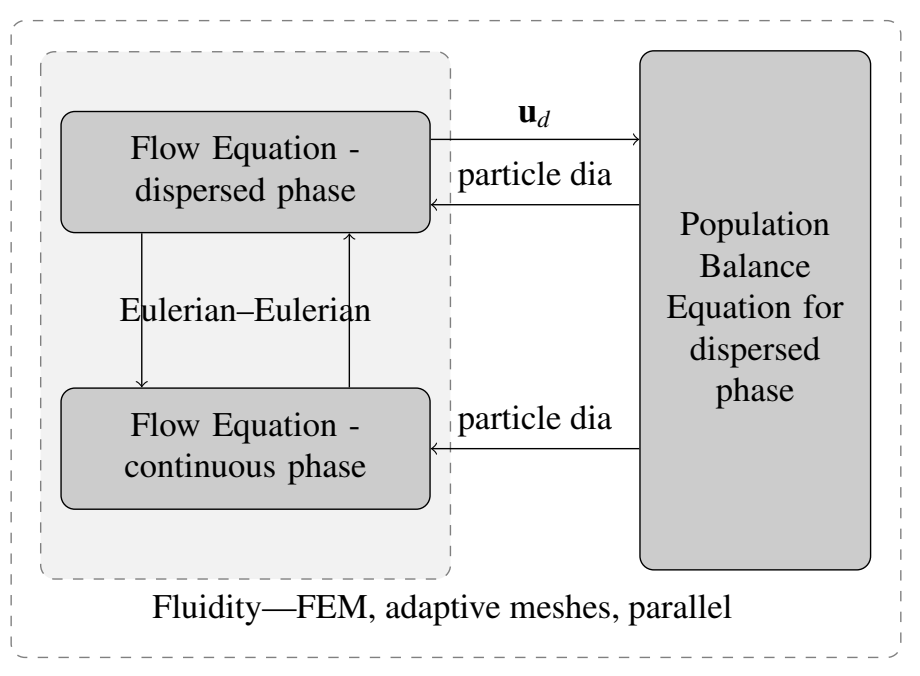

Figure 2: Diagram illustrating the approach used to model polydispersed flows in this work.

come from a continuous or a discontinuous function space. The subscripts CG, DG and CV refer to the continuous Galerkin, discontinuous Galerkin and the control volume discretisation methods, respectively. Differences between the three methods, in terms of the elements and the shape functions, are illustrated in Figure 1.

The $\mathrm{P} 1_{\mathrm{DG}}-\mathrm{P} 2$ velocity-pressure element pair was chosen in the simulations as this element pair ensured the LBB stability criterion (Gresho and Chan, 1988; Cotter et al., 2009). Furthermore, the use of discontiuous element shape functions allowed for the inversion of mass matrices locally, which preserved accuracy as opposed to mass lumping, which is carried out for continuous element shape functions.

\subsubsection{Solution method}

Figure 2 illustrates the approach that was followed in this work to model polydispersed flows. The flow equations and the population balance equation were coupled through the dispersed phase velocity, $\mathbf{u}_{d}$, and the dispersed phase diameter, $d$. The complete system was solved in Fluidity's finite element framework. This section outlines the implementation of the solution method that allowed for this coupling between the flow equations and PBE.

The algorithm that was implemented in Fluidity is detailed as follows:

Step 1. Calculate non-linear approximations for phase velocities, volume fractions and the Sauter mean diameter.

Step 2. Determine a tentative pressure field by either solving the Poisson equation for pressure (for the first time step) or using the value from previous time step.

Step 3. Predictor step: Compute intermediate velocity values for the two phases using the non-linear estimate of SMD from Step 1. 
Step 4. Corrector step: Correct the velocity values by making them divergence-free using the combined continuity equation (Equation (2)). Also, correct the tentative pressure by adding the pressure correction $\Delta p$.

Step 5. Solve population balance equation: Solve the DQMOM system of equations to get $w_{j}$ and $\langle\xi\rangle_{j}$, and thus get a new estimate for the SMD field.

Step 6. Repeat Steps (1)-(5) until the non-linear Picard iterations converge to give the field values $\mathbf{u}_{j}{ }^{n+1}, p^{n+1}, \alpha^{n+1}$ and $d_{32}{ }^{n+1}$ for the next time step $n+1$.

The above process was repeated until a steady state was reached or a desired number of time steps were completed.

\subsection{Mesh adaptivity}

Mesh adaptivity, also known in the literature as adaptive mesh refinement or grid adaptivity, is the name given to the method of modifying the mesh in a simulation to accurately predict the flow dynamics as time progresses.

In this work, fully-unstructured, anisotropic mesh adaptivity was performed to obtain optimum node placement for a given set of flow fields in the polydispersed problem. Mesh adaptivity was carried out in Fluidity using an interpolation-based method for the estimation of an a posteriori error metric (Babuška and Rheinboldt, 1978), which optimised the mesh iteratively until a given tolerance was met.

This section describes how mesh adaptivity is implemented in Fluidity. Although no part of any implementation of mesh adaptivity was carried out in this work, it seems important to the authors to explain how adaptivity works in this finite element framework, given that its application to polydispersed flows is the central theme of this paper.

The mesh adaptivity process in Fluidity can be understood as a sequence of three main steps (AMCG, 2015):

1. Metric estimation: a desired mesh is chosen.

2. Mesh generation: a mesh with the above characteristics is generated.

3. Field interpolation: all scalar, vector and tensor fields are transferred from the old mesh to the new adapted mesh.

These steps are explained in detail as follows.

\section{Metric estimation}

Mesh metric is an essential part of adaptivity responsible for measuring the topological characteristics of the mesh. Therefore, the first step in Fluidity's mesh adaptivity procedure is to estimate this metric based on the curvature of the field to be adapted to and a user-specified error bound. Essentially, the mesh metric is an estimate of the a posteriori error measure for a given solution field. This metric is a function of spatial position as well as direction and therefore, can generate nonhomogeneous and anisotropic meshes. (a)
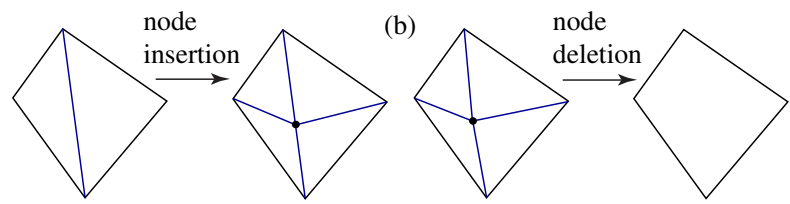

(c)

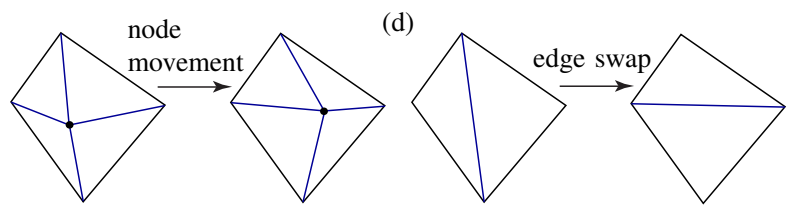

Figure 3: Mesh modification procedures invoked in two-dimensional adaptivity: (a) Node insertion, (b) node deletion, (c) node movement, and (d) edge swap (figure adapted from Piggott et al., 2009).

The curvature of a field, specified by its Hessian $H$, and the interpolation error bound $\epsilon$ can be used to calculate the Riemannian mesh metric $M$ as (Piggott et al., 2009):

$$
M=\frac{1}{\epsilon}|H| .
$$

The tensor $|H|$ here is a positive-definite metric obtained from the Hessian $H$ by taking an absolute value of all of its eigenvalues. In a sense, $|H|$ is a measure of the magnitude of the curvature and not its sign. Equation (23) can be understood as follows: tensor field $M$ allows for the placement of a high node density in regions of large field variation (curvature) or small (user-specified) interpolation error bound. The interpolation error $\epsilon$ can vary in space and time if required.

If the mesh needs to be adapted to more than one field, the individual field metrics, calculated from Equation (23), can be merged into one using the superposition method specified by Pain et al. (2001). Other constraints on the desired mesh such as maximum and minimum edge lengths, directionality constraints and aspect ratio bound can also be applied at this stage by superimposing more terms into the mesh metric and scaling its eigenvalues. Finally, before being sent to the mesh generation library, this metric is smoothed and scaled to avoid large variations in mesh sizes and to ensure that the number of nodes stays bounded.

\section{Mesh generation}

This is the second step in Fluidity's mesh adaptivity process. Once the mesh metric is successfully evaluated, the next step is to generate the new mesh through a sequence of local topological operations. Based on a variational functional that measures the quality of a generated element against the available metric, the mesh is optimised in steps using a set of mesh refinement operations. This element-by-element mesh optimisation is implemented like the Gauss-Seidel method where the old mesh is optimised iteratively and not regenerated completely. In twodimensions, as shown in Figure 3, the mesh modification steps include operations of the following kinds: node insertion (or 
edge splitting), node deletion, node movement and edge swapping (Piggott et al., 2009). These modification steps are carried out in succession, in Fluidity, until the functional reports an improvement is the generated mesh. Mesh generation in Fluidity is implemented using the mba2d algorithm in two-dimensions (Vasilevskii and Lipnikov, 1999) and using the method suggested by Pain et al. (2001) in three-dimensions.

The objective functional discussed above, responsible for gauging the mesh quality, works by locating the worst element in the mesh that then gets improved in the mesh optimisation subroutine. The whole process is repeated iteratively until a given tolerance is met. This functional operates in a non-Euclidean space where distances are calculated using the norm induced by the mesh metric $M$. The distance $r$ between two points (connected by the vector $v$ ) in the metric space is given by the following inner product:

$$
r=\left(v^{T} M v\right)^{1 / 2}
$$

i.e. the metric space is warped by $M$ in the same sense as it gets warped in Einstein's General Relativity (AMCG, 2015). An ideal mesh in this non-Euclidean space, that satisfies the metric $M$, consists of all equilateral triangles with unit edge lengths. Nevertheless, when transformed to the real space, the mesh becomes non-homogeneous as well as anisotropic. However, all mesh optimisation procedures are applied in the real space and the warped space is only used when calculating the functional value for the mesh under evaluation.

\section{Field interpolation}

Now that a mesh that conforms with the metric calculated in step 1 is generated, the third and the final step in Fluidity's mesh adaptivity procedure is the interpolation of all required fields from the old mesh on to the new mesh post adapt. Consistent interpolation is the easiest and most economical way to transfer information from the pre-adapt mesh to the post-adapt mesh. In the consistent interpolation method, field values are evaluated in the pre-adapt mesh corresponding to the new node locations, and these values are used as the coefficients for the finite element shape functions in the post-adapt state. This method is bounded and does not require the generation of a supermesh (Farrell et al., 2009). Galerkin projection method, on the other hand, is the preferred method in Fluidity when interpolating fields evaluated on discontinuous meshes. This method is also favoured when conservation of some field is essential but, unlike consistent interpolation, it requires generating the supermesh and is much more expensive.

To sum up, the adapted mesh in Fluidity is not constrained by the previous mesh in a strong sense and hence the mesh adaptivity procedure implemented by Fluidity is more flexible than any other $h r$-adaptive method (AMCG, 2015). More details on the implementation and usage of adaptive mesh refinement in Fluidity can be found in Pain et al. (2001), Piggott et al. (2009), the Fluidity Manual (AMCG, 2015) and references therein.

\section{Model verification}

A successful verification of the multiphase flow equations in Fluidity has been presented by Jacobs et al. (2013) using the method of manufactured solutions. In this section, verification of the solution of the population balance equation using DQMOM in Fluidity is presented. This verification exercise was performed for a spatially-homogeneous population balance equation that included particle aggregation and breakage.

McCoy and Madras (2003) presented an analytical solution for a spatially-homogeneous, transient population balance equation in their work, and this solution was used here to verify the DQMOM implementation in Fluidity. All equations in their work were developed with particle volume as the internal coordinate. Since a length-based formulation was used in the present work, the volume-based kernels of McCoy and Madras (2003) were converted to their corresponding length-based expressions. The length-based formulation of the analytical problem of McCoy and Madras (2003) along with its solution can be found in Appendix A.

\section{Table 1}

Kernel values for the two cases selected for verifying the PBE implementation in Fluidity.

\begin{tabular}{lcc}
\hline & Case 1 & Case 2 \\
\hline$\beta\left(\mathrm{m}^{3} \mathrm{~s}^{-1}\right)$ & $4.81 \times 10^{-12}$ & $4.81 \times 10^{-13}$ \\
$\tilde{s}\left(\mathrm{~m}^{-3} \mathrm{~s}^{-1}\right)$ & $2.0 \times 10^{12}$ & $8.0 \times 10^{13}$ \\
\hline
\end{tabular}

The verification simulations were performed for two sets of kernels, referred to as Case 1 and Case 2, in this paper. Table 1 lists the kernel values selected for the two cases. $\beta$ is the coalescence frequency, and the breakage frequency $a(\xi)$ was given by a power law relation as $a(\xi)=\tilde{s} \xi^{3}$. The first case was dominated by aggregation, whereas the latter was dominated by breakage. These kernel values were chosen such that the change in the SMD was between 40-60\% in a simulation time of $1 \mathrm{~s}$.

Figure 4 shows the plot of the Sauter mean diameter for the two cases. The evolution of SMD as a function of time is presented for the comparison of results obtained using Fluidity's DQMOM implementation and the analytical solution of McCoy and Madras (2003). There was an excellent match between the two and therefore the accuracy of the DQMOM implementation to solve PBE in Fluidity was confirmed.

Fluidity simulations in Figure 4 were run for $N=2, N$ being the number of quadrature points in the DQMOM approximaiton of the number density function (in Equation (17)). Although the results for $N=2$ were excellent, a comparison was performed with the results for $N=3$, as shown in Figure 5. As can be seen, there was no added benefit by introducing extra unknowns to the approximation of the NDF and $N=2$ was considered sufficient for the solution of the PBE. Since the number of initial moments that can be calculated using $N$ unknowns in the NDF 


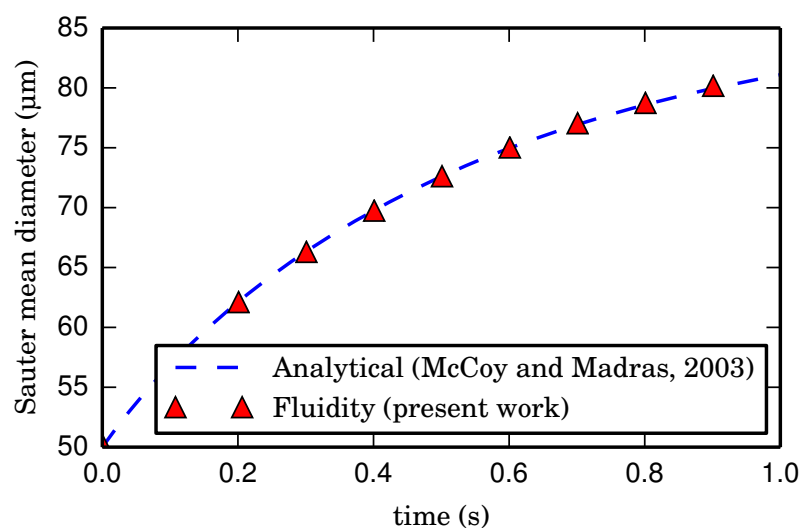

(a) Case 1

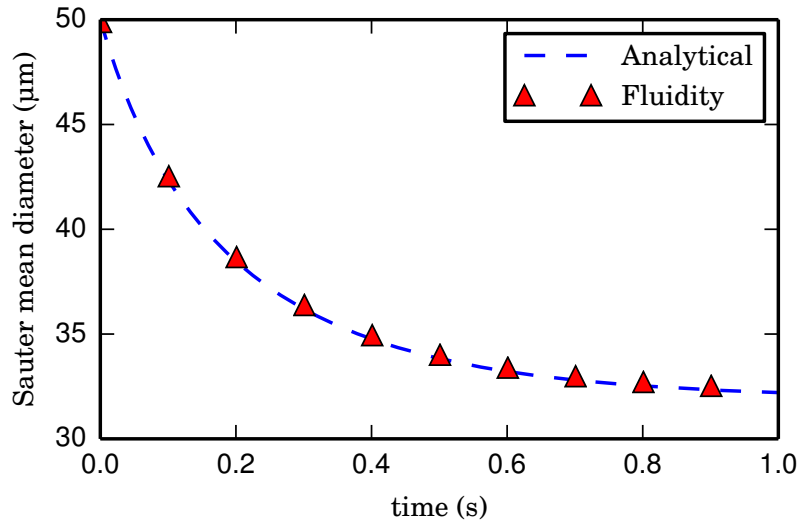

(b) Case 2

Figure 4: Verification of the DQMOM implementation for solving the population balance equation in Fluidity. Fluidity simulation results are compared against McCoy and Madras (2003)'s analytical solution. Results are presented for two cases: (a) Case 1: aggregation dominant, and (b) Case 2: breakage dominant.

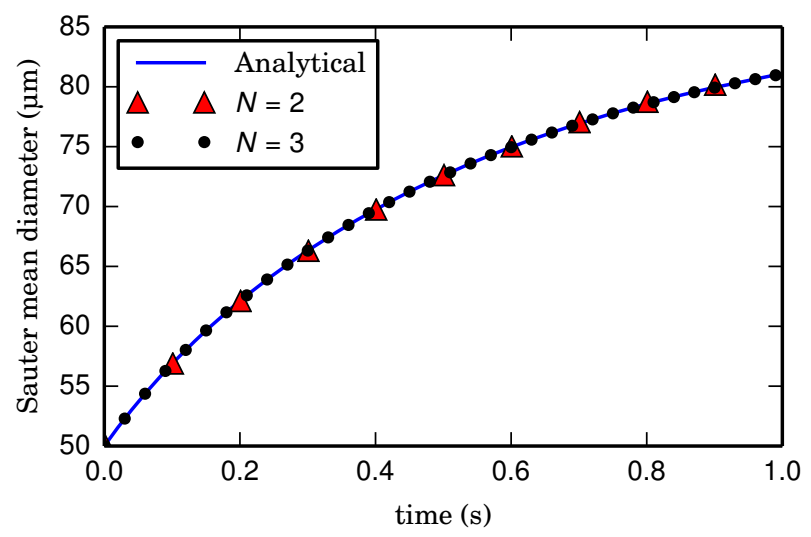

Figure 5: Effect of the number of unknowns used to approximate the number density function in DQMOM. Fluidity's PBE simulation results for $N=2$ and $N=3$ are shown and compared to the analytical solution of McCoy and Madras (2003). is $2 N$, a reference to ' $N$ unknowns of the NDF' may be used interchangeably with ' $2 N$ moments' in this paper.

\section{Results and discussions}

The results for the solution of a polydispersed two-phase flow problem using the finite element framework of Fluidity, employing fixed and adaptive meshes, are presented in this section. The flow equations, coupled with the population balance equation, were solved using the hybrid FE-CV method, as explained in Section 2.3.1.

A two-phase water-in-oil emulsion was simulated in a twodimensional Backward Facing Step (BFS) geometry, as shown in Figure 6, to illustrate the effectiveness of the solution method developed in this work. Water droplets were dispersed in a continuous oil phase where they could coalesce and break. There was no gravitational force on the phases and they moved purely under inertia.

Silva et al. (2008) and Abbasi and Arastoopour (2013) also used a similar arrangement (to demonstrate the use of population balances for modelling polydispersed flow problems) due to the simplicity and popularity of this benchmarking flow problem. The backward facing step contains a region of primary recirculation with large flow gradients, which can be exploited for studying various flow phenomena. The problem setup in this work differed from that of Silva et al. (2008) in the dimensions of the BFS considered and in the breakage and aggregation rates used for modelling the water droplet population evolution. The BFS geometry considered in this paper is the same as the one used by Armaly et al. (1983) in their classic benchmark experiments, except for the third dimension.

As mentioned in Section 2.2, the PBE in this work was formulated for three-dimensional dispersed particles. Hence the problem solved in this paper physically corresponded to a 'thin' 3D backward facing step where variations in the third dimension were 'small'. The flow fields like velocity, pressure and volume fraction depended on the $\mathrm{x}$ and $\mathrm{y}$ coordinates only. The particles still were modelled as three-dimensional in the PBE source terms, i.e. when two water drops coalesced, the resulting drop volume was the sum of the two contributing drops.

As a first step, simulation results for a monodispersed backward facing step (with constant water drop diameter) are presented to obtain an understanding of this two-phase flow problem. This is followed by polydispersed flow simulation results, which were benchmarked against a commercial CFD software. Finally, simulation results using fully-unstructured, anisotropic, adaptive mesh refinement are presented and their advantages over fixed meshes are detailed at the end of this section.

\subsection{Monodispersed backward facing step}

The backward facing step problem shown in Figure 6 was initially solved for a constant water drop diameter of $50 \mu \mathrm{m}$. The 


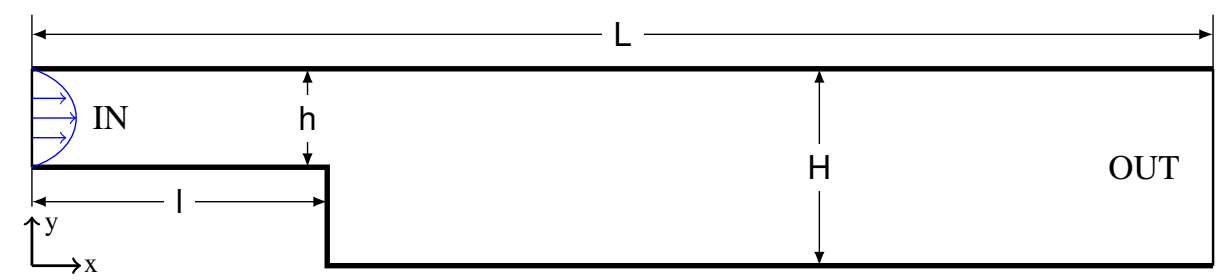

Figure 6: Two-dimensional backward facing step geometry used for modelling monodispersed and polydispersed water-in-oil emulsion. $L=260, l=60, h=5.2$ and $H=10.1$. All dimensions in mm (figure not to scale).

initial and boundary conditions used for this simulation setup in Fluidity are shown in Tables 2 and 3, respectively. The $\mathrm{x}$ component of the inlet velocity for both phases was parabolic with a mean value of $0.56 \mathrm{~m} \mathrm{~s}^{-1}$. The value of the physical parameters chosen for this simulation are detailed in Table 4.

\section{Table 2}

Initial conditions for the monodispersed backward facing step simulation.

\begin{tabular}{lc}
\hline Field name & Initial value \\
\hline Oil velocity $\left(\mathrm{m} \mathrm{s}^{-1}\right)$ & $(0,0)$ \\
Water velocity $\left(\mathrm{m} \mathrm{s}^{-1}\right)$ & $(0,0)$ \\
Water volume fraction & 0.1 \\
\hline
\end{tabular}

Table 5 shows a list of the numerical parameters that were used in the setup of this monodispersed BFS simulation. A fully unstructured mesh with a characteristic mesh size of $0.5 \mathrm{~mm}$ was used. Since the field gradients were large near the step of the BFS, the mesh was refined to a characteristic length of a quarter of the usual size in the step region (i.e. $0.125 \mathrm{~mm}$ ). A fixed time step of $1.0 \times 10^{-5} \mathrm{~s}$ was chosen for this simulation, which ensured that the Courant-Friedrichs-Lewy (CFL) number was bounded below one all the time.

Methods used for discretising the flow equations for the two phases are shown in Table 6. As can be seen from this table, a control volume discretisation scheme was chosen for the volume fraction equation due to its conservative nature as opposed to the continuous Galerkin or discontinuous Galerkin discretisations. Ensuring the conservation of volume fraction in multiphase flows is very important, especially since it is strongly coupled to the momentum, energy and turbulence equations. As discussed in Section 2.3.1, a hybrid FE-CV scheme was used to model the problem in this work due to its advantages over pure finite element or control volume approaches.

The advection terms in the momentum equations were discretised using a non-conservative form in this case. The final linear systems obtained for the momentum equations were solved using the Generalised Minimal RESidual method (GMRES) in conjunction with the Successive Overrelaxation (SOR) preconditioner.

For the BFS simulations in this paper, the continuous phase Reynolds number was 524, which was safely in the laminar flow regime as per Armaly et al. (1983). The definition of the continuous phase Reynolds number used here is the same as that used by Armaly et al. (1983):

$$
R e_{c}=\frac{\rho_{c}\left|\mathbf{u}_{c}\right| D}{\mu_{c}},
$$

where $\mathbf{u}_{c}$ is the continuous phase (oil) velocity, and $D=2 h$ with $h$ being the height of the small channel (see Figure 6).

The Schiller-Naumann drag force correlation (Equation (7)) used in this work assumes rigid spherical dispersed particles (Schiller and Naumann, 1935). Although this drag force correlation was originally developed for solid dispersed particles, Ishii and Zuber (1979) stated that the drag for a dispersed fluid phase can also be estimated using the same correlation as long as the fluid particles are less than some specified size (beyond which the distortion and irregular effects become noticeable). The polydispersed water droplets modelled in this work were less than $85 \mu \mathrm{m}$ in size, for which the Schiller-Naumann drag force correlation was applicable. Roudsari et al. (2012) successfully used the Schiller-Naumann drag correlation for modelling water-in-oil emulsion with water droplets more than $100 \mu \mathrm{m}$ in size. If the dispersed drops become large, their shape may deviate from spherical and the Schiller-Naumann drag force correlation and the Sauter mean diameter may not be applicable to accurately predict the interphase drag force. The Schiller-Naumann interphase drag coefficient has been shown to fit the standard drag curve well for Reynolds number values less than 800 with only a 5 percent deviation (Clift et al., 1978). Although these comparisons have been made for a single dispersed sphere, others have successfully extended the use of this drag coefficient for multiple dispersed particles in flow (Buwa and Ranade, 2002; Tabib et al., 2008; Silva et al., 2008).

Results for the monodispersed BFS can be seen from Figure 7 , which shows the water velocity contours at four time instances. Development of the primary and the secondary recirculation zones is apparent from these contours and it can also be seen that their length becomes stable by $t=1 \mathrm{~s}$. Hence all subsequent (polydispersed flow) simulations have been presented for a maximum simulation time of $1 \mathrm{~s}$ as no new transient phenomenon was seen beyond this time.

In addition to obtaining an understanding of the flow characteristics, the monodispersed BFS simulation was also carried out to obtain a set of discretisations and optimum numerical parameter values. These were then used in the simulations of the polydispersed BFS, the results for which are presented in the subsequent section. 
Table 3

Boundary conditions for the monodispersed backward facing step simulation. $n$ here refers to the normal coordinate.

\begin{tabular}{lllll}
\hline Boundary & Oil velocity & Water velocity & Pressure & Water volume fraction \\
\hline Inlet & $u_{c}=$ parabolic, $\mathrm{v}_{c}=0$ & $u_{d}=$ parabolic, $\mathrm{v}_{d}=0$ & $\frac{\partial p}{\partial n}=0$ & 0.1 \\
Walls & no slip (weak) & no slip (weak) & $\frac{\partial p}{\partial n}=0$ & no flux \\
Outlet & $\frac{\partial u_{c}}{\partial n}=\frac{\partial \mathrm{v}_{c}}{\partial n}=0$ & $\frac{\partial u_{d}}{\partial n}=\frac{\partial \mathrm{v}_{d}}{\partial n}=0$ & $p=0$ & $\frac{\partial \alpha_{d}}{\partial n}=0$ \\
\hline
\end{tabular}
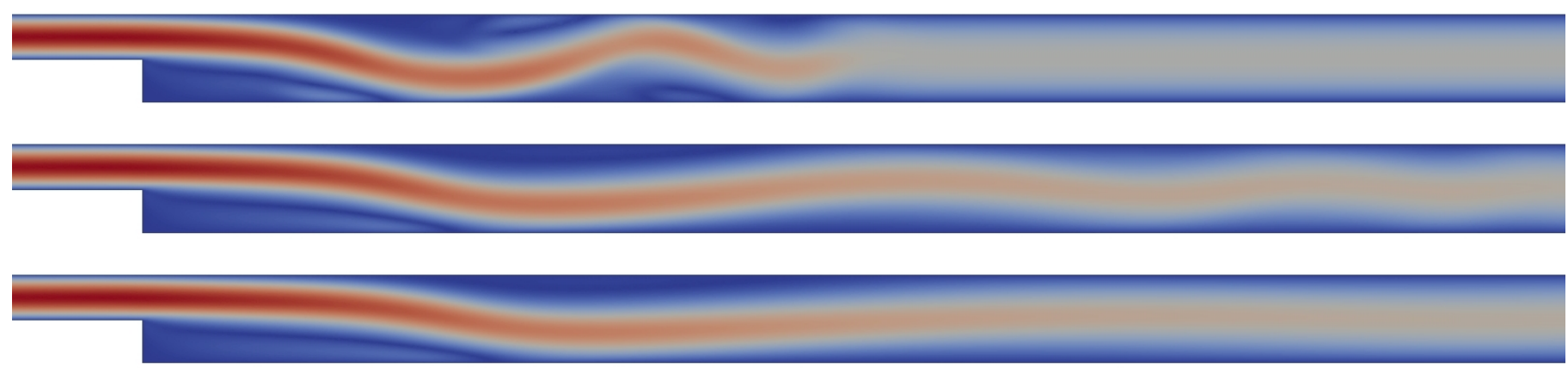

secondary recirculation zone
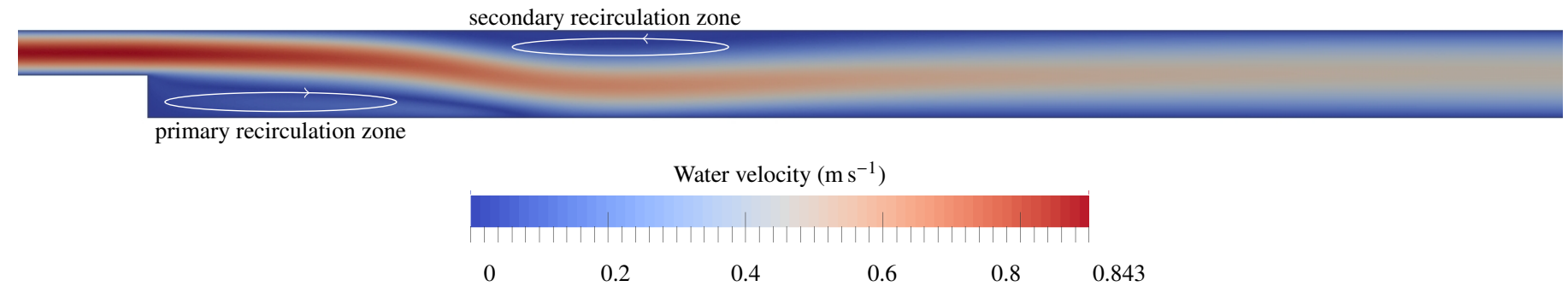

Figure 7: Water velocity contours at times (top to bottom) $0.25 \mathrm{~s}, 0.5 \mathrm{~s}, 0.75 \mathrm{~s}$ and $1.0 \mathrm{~s}$ for a monodispersed backward facing step with $50 \mu \mathrm{m}$ water droplets.

\section{Table 4}

Physical parameters used in the monodispersed backward facing step simulation.

\begin{tabular}{lc}
\hline Physical parameter & Value \\
\hline Oil density $\left(\mathrm{kg} \mathrm{m}^{-3}\right)$ & 900 \\
Water density $\left(\mathrm{kg} \mathrm{m}^{-3}\right)$ & 1000 \\
Oil viscosity $(\mathrm{Pa} \cdot \mathrm{s})$ & 0.01 \\
Water viscosity $(\mathrm{Pa} \cdot \mathrm{s})$ & 0.001 \\
\hline
\end{tabular}

\section{Table 5}

Numerical parameters used in the monodispersed backward facing step simulation.

\begin{tabular}{lc}
\hline Numerical parameter & Value \\
\hline Mesh size $(\mathrm{mm})$ & 0.5 \\
Time step $\Delta t(\mathrm{~s})$ & $10^{-5}$ \\
Overall simulation time $(\mathrm{s})$ & 1.0 \\
Number of Picard iterations & 2 \\
Tolerance for Picard iterations $\left(L^{2}\right.$-norm $)$ & $10^{-12}$ \\
\hline
\end{tabular}

\subsection{Polydispersed backward facing step}

A polydispersed water-in-oil emulsion in a backward facing step was simulated by coupling the flow equations with the population balance equation, as described in Section 2.3.2. In this work, the direct quadrature method of moments (Marchisio and Fox, 2005), that was implemented in Fluidity, was used to solve the population balance equation in conjunction with the Eulerian-Eulerian method for the multiphase flow equations.

The initial and boundary conditions used for the flow equations in this model were the same as the ones used for the monodispersed BFS (Section 4.1). For the population balance equation, the initial conditions were specified for the first four moments of the droplet number density function. These initial moment values were based on an initial volume fraction of 0.1 and an initial Sauter mean diameter of $50 \mu \mathrm{m}$ for the dispersed (water) phase. For spherical particles, the volume fraction of the dispersed phase, $\alpha_{d}$, is related to the third moment of the NDF as:

$$
m_{3}=\alpha_{d}\left(\frac{6}{\pi}\right) .
$$

Assuming the dispersed particles to be spherical, Equation (26) 


\section{Table 6}

Numerical discretisations used for the flow equations for oil and water phases in the monodispersed backward facing step simulation.

\begin{tabular}{lll}
\hline & Oil & Water \\
\hline Velocity & P1 Discontinuous Galerkin (DG) & P1 DG \\
& Upwind for advection, Bassi-Rebay for vis- & Upwind for advection, Bassi-Rebay for vis- \\
& cous terms (Bassi and Rebay, 1997) & cous terms \\
Pressure & - & P2 continuous Galerkin \\
Time & $\theta=0.5$ (Crank-Nicolson) & Crank-Nicolson \\
Volume fraction & - & P0 CV with first-order upwind \\
\hline
\end{tabular}

\section{Table 7}

Initial conditions for the moments $(m)$ of the number density function used in the population balance equation for simulating the polydispersed water-in-oil emulsion in a backward facing step.

\begin{tabular}{lc}
\hline$m_{0}$ & $2.077 \times 10^{12}$ \\
$m_{1}$ & $8.372 \times 10^{7}$ \\
$m_{2}$ & $3.820 \times 10^{3}$ \\
$m_{3}$ & $1.910 \times 10^{-1}$ \\
\hline
\end{tabular}

and Equation (16) were used to evaluate the second and third moments in terms of the initial conditions on volume fraction and SMD. All other moments (i.e. $m_{0}$ and $m_{1}$ here) were calculated in terms of $m_{2}$ and $m_{3}$ using the moments of the initial number density function of McCoy and Madras (2003) (Equation (A.1)). The first four moments used as initial conditions in this work are listed in Table 7. The corresponding initial values for the solution fields (weights and weighted abscissas) were calculated from these moments in Fluidity using the ProductDifference (PD) algorithm (Gordon, 1968). See Appendix A (Equations (A.11)-(A.14)) for the formulation of the lengthbased moments of the initial distribution of McCoy and Madras (2003).

Boundary Conditions (BCs) used for the population balance equation fields can be seen in Table 8 . The BCs at the inlet were exactly the same as the initial conditions, i.e. the set of BCs for weights and weighted abscissas at the inlet given in Table 8 correspond to the same set of moments specified in Table 7 .

A P0 Control Volume (CV) discretisation with first-order upwinding for the advection terms was used for the weights and the weighted abscissas in the PBE. Time derivatives in the PBE field equations were discretised using the Crank-Nicolson scheme $(\theta=0.5)$. All other discretisations for the remaining fields were the same as the ones used in the monodispersed BFS simulation, given in Section 4.1.

Four unknowns (i.e. $N=2$ in Equation (17)) were chosen to approximate the water droplet number density function in this simulation as the SMD could be estimated with a good accuracy without adding any more unknowns. Figure 5 in Section 3 presents a justification for the adequacy of using $N=2$ in the DQMOM implementation in this work.
The source terms for the population balance equation, describing birth and death of drops due to coalescence and breakage, were exactly the same as specified in the Section 3 . The simulations were run for two sets of breakage and coalescence kernels, which are referred to as Case 1 and Case 2. Case 1 was dominated by aggregation, whereas Case 2 corresponded to a breakage dominant case. The kernel values can be found in Table 1 in Section 3.

It must be clarified here that the breakage and aggregation kernels had no physical significance in this hypothetical BFS flow problem, and were implemented solely to demonstrate the strengths of Fluidity in solving polydispersed multiphase problems. However, they did make the process of code verification easy because of the existence of an analytical solution for the spatially-homogeneous case (see Section 3).

Figure 8 shows the surface plots of the Sauter mean diameter for water drops at four different simulation times for Case 1. It can be seen from this figure that the recirculation zones, where drops had more time to coalesce, were the regions having higher SMD. A similar behaviour was seen for the breakage dominated case (Case 2) where recirculation zones corresponded to the regions of smaller drop diameters as illustrated in Figure 9.

\subsubsection{Mesh Convergence}

The polydispersed BFS simulation was run for three different fixed meshes, for Case 1, to study mesh convergence in this problem. Details of the three fixed meshes, referred to as Coarse, Medium and Fine mesh, can be found in Table 9. A very fine mesh simulation, called the Superfine mesh, was also run with a total of 147,308 nodes and a characteristic mesh size of $0.2 \mathrm{~mm}$. This Superfine mesh was selected as a proxy for the exact solution to the problem, for the purpose of calculating errors in the solutions produced by the other three meshes. All fixed meshes were generated using the open-source mesh generation software Gmsh (Geuzaine and Remacle, 2009). Figure 10 shows a part of the Coarse mesh that was used in the simulation. The unstructured nature of the mesh can be seen clearly along with the refined elements near the step. The mesh elements near the step were four times smaller than usual to capture the strong field variations in that region. All other fixed meshes, except for their finer element size, were similar to the 


\section{Table 8}

Boundary Conditions (BCs) for the population balance equation solution fields used in the polydispersed backward facing step simulation. BCs are presented for the weights $\left(w_{1}\right.$ and $\left.w_{2}\right)$ and the weighted abscissas $\left(\varsigma_{1}\right.$ and $\left.\varsigma_{2}\right) . n$ here refers to the normal coordinate

\begin{tabular}{lcccc}
\hline Boundary & $\boldsymbol{w}_{\mathbf{1}}$ & $\boldsymbol{w}_{\mathbf{2}}$ & $\boldsymbol{S}_{\mathbf{1}}$ & $\boldsymbol{\zeta}_{\mathbf{2}}$ \\
\hline Inlet & $1.125 \times 10^{12}$ & $9.514 \times 10^{11}$ & $3.020 \times 10^{7}$ & $5.351 \times 10^{7}$ \\
Walls & no flux & no flux & no flux & no flux \\
Outlet & $\frac{\partial w_{1}}{\partial n}=0$ & $\frac{\partial w_{2}}{\partial n}=0$ & $\frac{\partial \varsigma_{1}}{\partial n}=0$ & $\frac{\partial \varsigma_{2}}{\partial n}=0$ \\
\hline
\end{tabular}
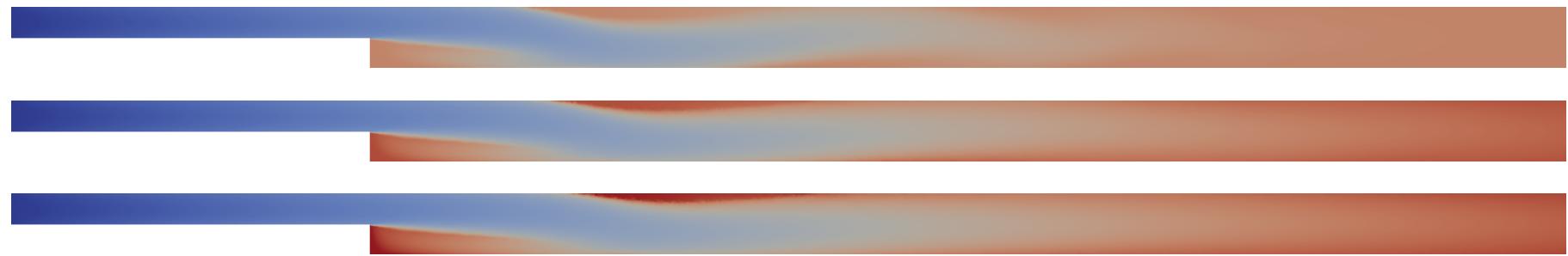

Water Sauter mean diameter $(\mu \mathrm{m})$

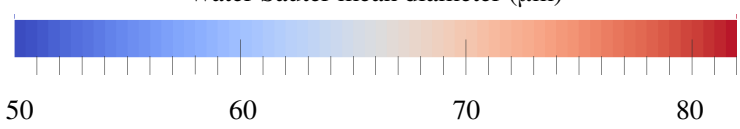

Figure 8: Sauter mean diameter of water drops for the aggregation dominated case (Case 1) at four different times: (top to bottom) $0.25 \mathrm{~s}, 0.5 \mathrm{~s}, 0.75 \mathrm{~s}$ and $1.0 \mathrm{~s}$. The primary and the secondary recirculation zones for the SMD can be seen.

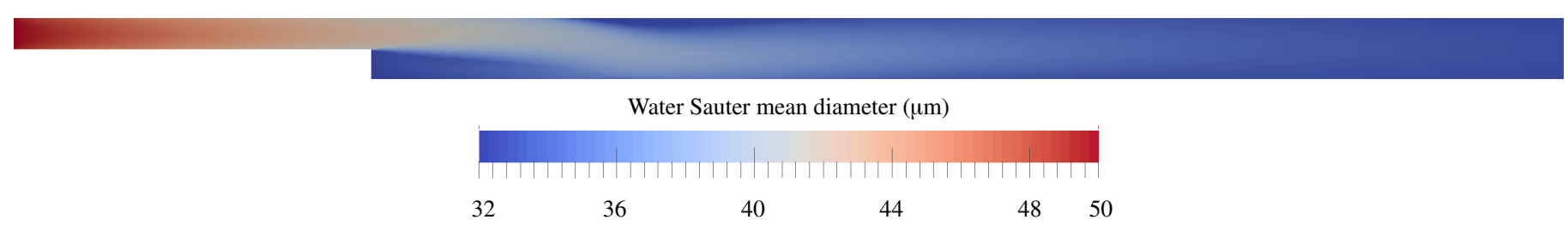

Figure 9: Sauter mean diameter of water drops for the breakage dominated case (Case 2) at four different times: (top to bottom) $0.25 \mathrm{~s}, 0.5 \mathrm{~s}, 0.75 \mathrm{~s}$ and $1.0 \mathrm{~s}$. The primary and the secondary recirculation zones for the SMD can be seen.

\section{Table 9}

Details of the three fixed meshes used for the mesh convergence analysis of the solution of polydispersed backward facing step in Fluidity.

\begin{tabular}{lcc}
\hline Mesh & Mesh nodes & Mesh size $(\mathbf{m m})$ \\
\hline Coarse & 19,825 & 0.55 \\
Medium & 24,100 & 0.5 \\
Fine & 65,967 & 0.3 \\
\hline
\end{tabular}

Coarse mesh.

Jacobs et al. (2013) showed a second order convergence for both velocity and pressure in multiphase flow simulations for the $\mathrm{P} 1_{\mathrm{DG}}-\mathrm{P} 2$ element pair in Fluidity. The same velocitypressure element pair was used here, with an additional population balance equation that was discretised using the control volume scheme. To study the mesh convergence of the population balance equation, the fixed mesh simulations were run up to $1 \mathrm{~s}$ and the error in the Sauter mean diameter of water drops was plotted as a function of mesh size, as illustrated in Figure 11. The order of convergence obtained was close to the ex- 


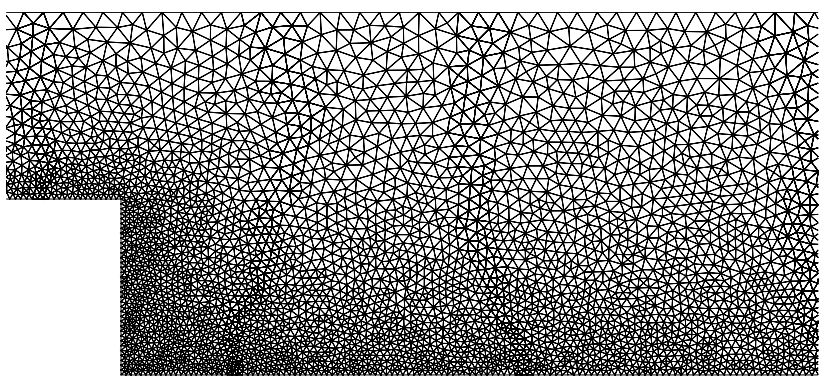

Figure 10: Fixed mesh used in the simulation of the polydispersed water-in-oil emulsion. This is the Coarse mesh with a characteristic mesh size of $0.55 \mathrm{~mm}$. All other fixed meshes were similar to this Coarse mesh except for a different mesh size.

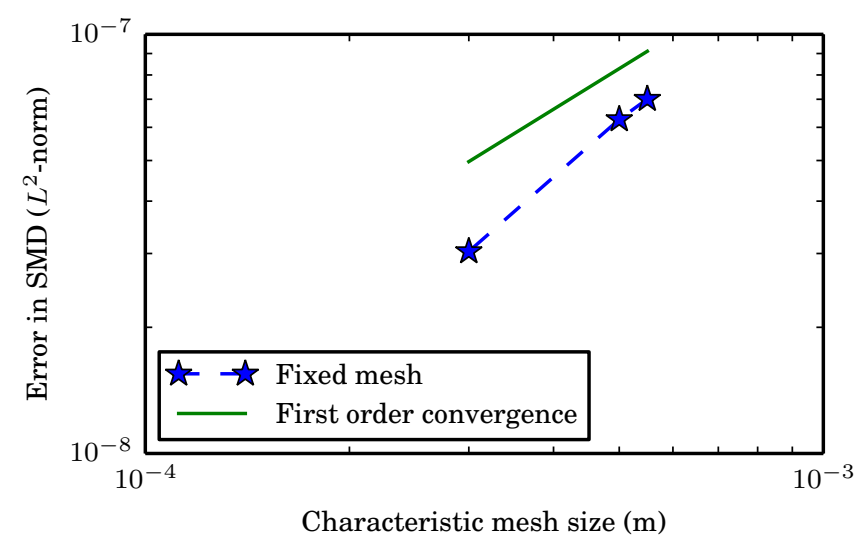

Figure 11: Convergence plot for the Sauter mean diameter of water drops. The population balance equation converged to first order as expected.

pected first-order convergence for the control volume discretisation method.

To compare the results produced by the three fixed meshes, the Sauter mean diameter of water drops was plotted along a vertical line $5 \mathrm{~mm}$ from the step, as shown in Figure 12. The results obtained for the three fixed meshes were close, as illustrated in Figure 13, and therefore the Medium mesh was chosen for all subsequent fixed mesh simulations.

\subsubsection{Benchmarking}

In order to gauge the accuracy of the results obtained using Fluidity, ANSYS Fluent was used as benchmark against which the results in the present work were compared. Fluent has been used by many, including Marchisio et al. (2004), Zucca et al. (2006) and Buffo et al. (2012) to solve the population balance

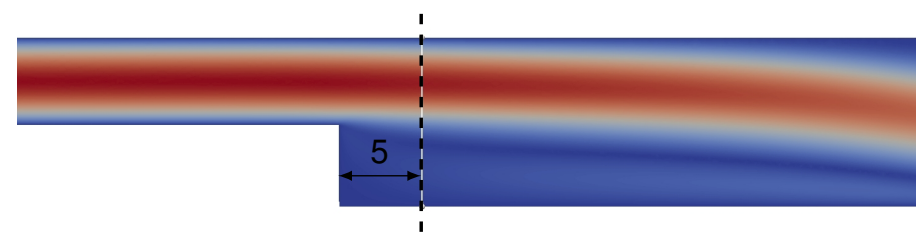

Figure 12: Vertical line at a distance of $5 \mathrm{~mm}$ from the step along which the Sauter mean diameter values have been plotted for the water drops.

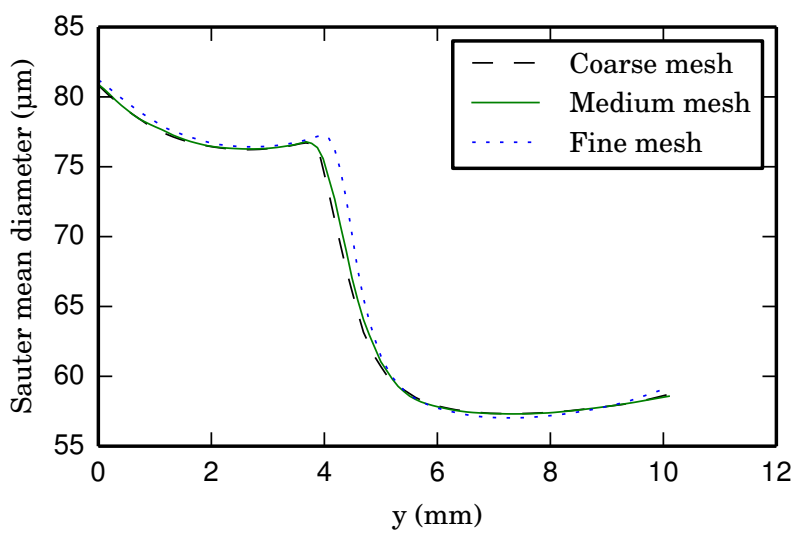

Figure 13: Sauter mean diameter of the water drops plotted along a vertical line $5 \mathrm{~mm}$ from the step of the BFS. Results for the three fixed mesh simulations are compared here.

equation using QMOM and DQMOM. Hence, it was chosen as a benchmark to compare the results obtained for the simulation of this hypothetical polydispersed water-in-oil emulsion in the backward facing step in this work. The aggregation and breakage kernels used to model drop coalescence and breakage were completely hypothetical in this work and do not correspond to a physical scenario. The current problem was chosen to demonstrate the effectiveness of the framework developed as a part of this work to model polydispersed multiphase flows.

The Medium mesh case was simulated in Fluent with the configuration options listed in Table 10. All Fluent simulations were run using ANSYS Academic Research, Release 15.0 .

A comparison of the plot of the Sauter mean diameter of water drops along a vertical line $5 \mathrm{~mm}$ from the step, obtained using Fluidity and ANSYS Fluent, is shown in Figure 14. Note that a good match between Fluent and Fluidity results can be seen for Cases 1 and 2 in Figures 14(a, b). It must be stressed that QMOM (McGraw, 1997) was used for solving the population balance equation in Fluent, whereas Fluidity used DQMOM for the same. Also, Fluent uses the finite volume method instead of the finite element method used by Fluidity, and the numerical discretisations used for the two were also different. These differences can possibly explain the discrepancies between the two solutions seen in Figures 14(a, b).

\subsubsection{Discretisation schemes for the population balance equa- tion}

A CV+first-order upwind scheme was used to discretise the population balance equation in this work and all the results are presented with this discretisation. This was due to the conservative and robust nature of this scheme. Two other schemes were also tested for discretising the PBE and a comparison of the results obtained is a topic of discussion for this section. Figure 15 shows plots of the SMD along a vertical line $5 \mathrm{~mm}$ from the step for Case 1, for the three discretisation schemes. It is evident that the Continuous Galerkin (CG) scheme with Streamwise Up- 
Table 10

Simulation configuration settings for Fluent used for modelling the polydispersed water-in-oil emulsion in a backward facing step.

\begin{tabular}{|c|c|c|}
\hline \multirow[t]{2}{*}{ Solver } & \multicolumn{2}{|l|}{ pressure-based } \\
\hline & transient & \\
\hline \multirow[t]{8}{*}{ Model } & multiphase & 2 Eulerian phases \\
\hline & & implicit \\
\hline & & Schiller-Naumann drag \\
\hline & & $\begin{array}{l}\text { drop diameter: Sauter mean diame- } \\
\text { ter }\end{array}$ \\
\hline & viscous & laminar \\
\hline & population balance & QMOM \\
\hline & & 4 moments \\
\hline & & $\begin{array}{l}\text { UDFs for breakage and aggregation } \\
\text { kernels }\end{array}$ \\
\hline \multirow[t]{5}{*}{ Boundary conditions } & walls & stationary walls \\
\hline & inlet & $\begin{array}{l}\text { velocity: UDF for parabolic veloc- } \\
\text { ity }\end{array}$ \\
\hline & & water volume fraction $=0.1$ \\
\hline & & water moments specified \\
\hline & outlet & pressure outlet \\
\hline \multirow[t]{6}{*}{ Solution methods } & P-V coupling & coupled scheme \\
\hline & spatial discretisation & gradients: least squares cell based \\
\hline & & momentum: third-order MUSCL \\
\hline & & volume fraction: first-order upwind \\
\hline & & moments: first-order upwind \\
\hline & transient discretisation & first-urder implicit \\
\hline \multirow[t]{4}{*}{ Relaxation parameters } & momentum & 0.75 \\
\hline & pressure & 0.75 \\
\hline & volume fraction & 0.5 \\
\hline & moments & 0.5 \\
\hline
\end{tabular}




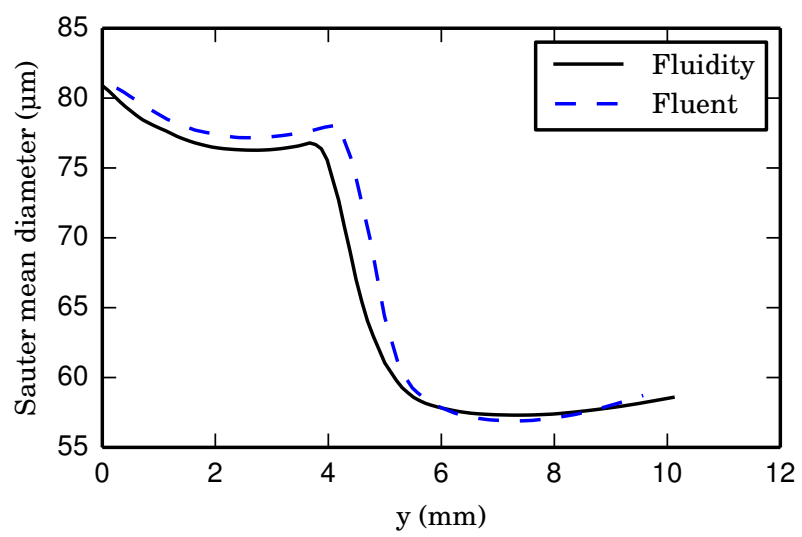

(a) Case 1

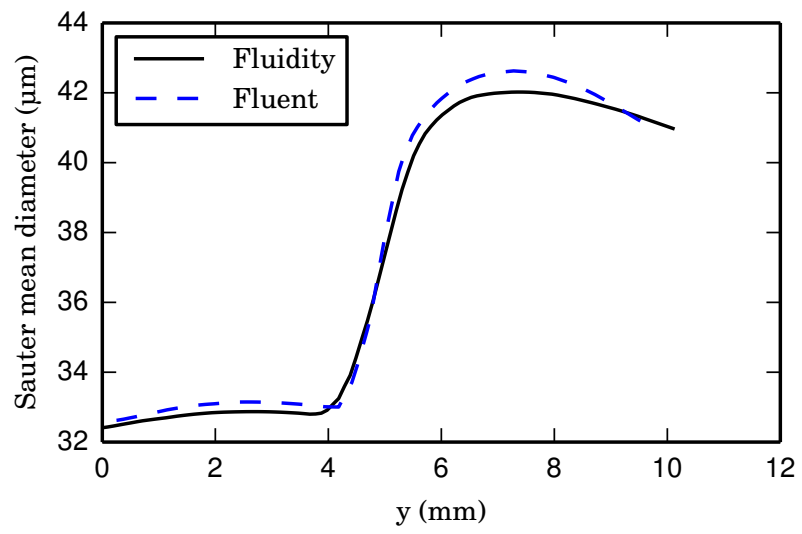

(b) Case 2

Figure 14: Fluidity results benchmarked against ANSYS Fluent results for: (a) the aggregation dominated case (Case 1), and (b) the breakage dominated case (Case 2). Sauter mean diameter of the water drops has been plotted along the vertical line shown in Figure 12, for the two solvers.

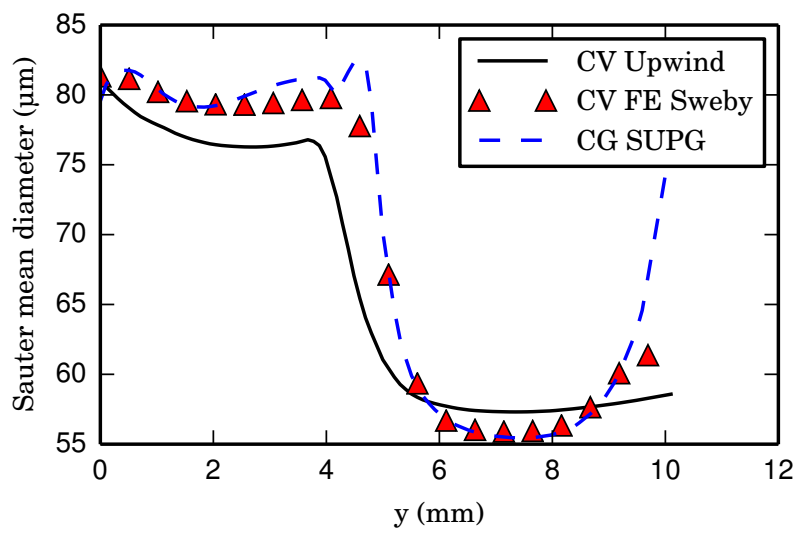

Figure 15: Comparison of different discretisation schemes tried for the population balance equation. Plot of the Sauter mean diameter along a vertical line $5 \mathrm{~mm}$ from the step has been shown for the aggregation dominant case (Case 1) at $t=1 \mathrm{~s}$. Continuous Galerkin (CG) discretisation method with a Streamwise Upwind Petrov Galerkin (SUPG) stabilisation showed the most accurate result with minimum diffusivity amongst all discretisation schemes that were tested. Despite this, the CV scheme, with first-order upwind discretisation for the convective terms, was chosen for all polydispersed flow simulations due to its conservative and stable nature, unlike other schemes.

wind Petrov Galerkin (SUPG) stabilisation for the convective terms was the least diffusive of all, as seen from the slope of the line between $y=4 \mathrm{~mm}$ and $y=6 \mathrm{~mm}$, and hence was able to resolve the primary recirculation boundary most accurately. It was also able to resolve the top boundary layer, as displayed by the SMD value around $y=10 \mathrm{~mm}$. On the other hand, the Control Volume $(\mathrm{CV})$ scheme with first-order upwind discretisation for the convection terms was most diffusive. Somewhere in the middle was the CV scheme with a finite element discretisation for convective terms, with a Sweby limiter (Sweby, 1984), as shown in Figure 15.

Figure 16 shows the surface plots for the water SMD at $t=1 \mathrm{~s}$ for the three schemes. Accuracy of the $\mathrm{CG}$ and the $\mathrm{CV}+\mathrm{FE}$ schemes is visually apparent, through the better resolution of the two recirculation zones, over the $\mathrm{CV}+$ first-order upwind scheme. Despite the advantages of the two accurate schemes, the $\mathrm{CV}+$ first-order upwind scheme was chosen for all simulations due to its conservative and monotonic nature, which ensured that the moments were conserved and the method was unconditionally stable.

Results for the polydispersed multiphase flow simulations were presented in this section and the effectiveness of the finite element framework of Fluidity in modelling such flows was demonstrated. Offering an alternative over the other (very few) available commercial and non-commercial flow solvers that are able to deal with multiphase polydispersed flows, Fluidity's FE-CV hybrid approach was shown to be able to gather the best of both worlds - finite element and control volume-and simulate such flows with good accuracy.

The framework of Fluidity is parallelised and all fixed meshes were decomposed and distributed among different processors to be able to solve the simulations quickly. All simulations presented here were parallelised to 8 cores. To make the simu- 


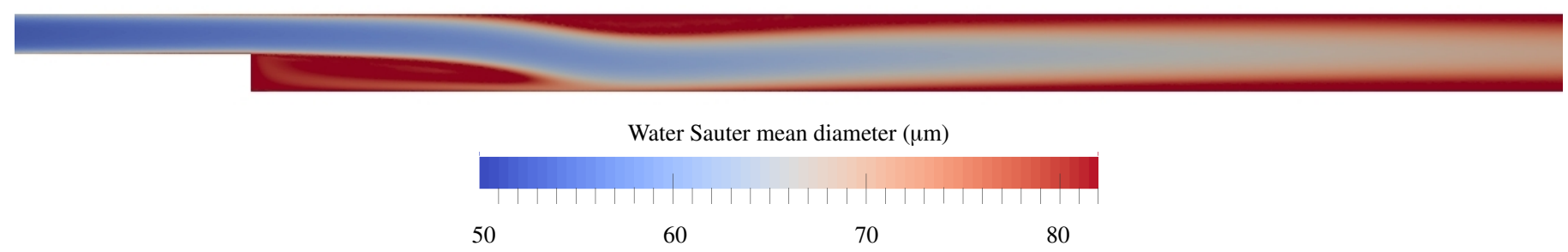

Figure 16: Sauter mean diameter of water drops for Case 1 at $t=1 \mathrm{~s}$ for three different discretisation schemes for the population balance equation. From top to bottom: CV+first-order upwind, CV+finite element (Sweby limiter) and CG+SUPG stabilisation. CG+SUPG discretisation was able to resolve the two recirculation zones better than the two other schemes. Despite this, the control volume scheme, with first-order upwind discretisation for the convective terms, was chosen for all polydispersed flow simulations due to its conservative and stable nature, unlike other schemes.

lation process more efficient, mesh adaptivity, as discussed in Section 2.4, was also introduced in the simulations and the results demonstrating the excellent improvement obtained using this process are discussed in the next section.

\subsection{Mesh adaptivity}

The transient flow field results shown in the previous section (Section 4.2) were all for a fixed mesh, which did not change with time. Such a fixed mesh can rarely be optimum for a dynamic problem like the one under consideration. Optimum mesh node positioning cannot be decided in advance as the flow features are changing continuously due to the inherent complex dynamics of the problem. Mesh adaptivity offers a way to handle this issue by adapting the mesh in time and hence, was applied to the polydispersed water-in-oil emulsion flow problem in this work. This section presents and analyses the results for an adaptive mesh simulation of the polydispersed flow and compares the solution errors and runtimes to the fixed mesh simulations presented in the previous section.

An adaptive mesh simulation was set up for the aggregation dominated (Case 1) water-in-oil emulsion problem in a BFS, where the mesh was adapted to the water droplet size and velocity. The reason for this choice was that water droplet size and velocity were the fields of interest in this work and the other fields were observed to vary similar to these two in space. At first, the mesh was adapted to the water Sauter mean diameter only, as this was the most important scalar field in this polydispersed flow problem. However, it was realised that the mesh adapted to the water SMD alone was not able to resolve the strong variations in water velocity in the y-direction when the mesh became excessively coarse in regions away from the recirculation zone boundaries. The increased error in flow velocity associated with the coarse mesh in these regions required the inclusion of the water velocity in the list of fields the mesh was adapted to. Also, a bound was set on the maximum mesh resolution to prevent it from going excessively coarse in "noncritical" regions.

The interpolation error bound values used for the calculation of the error metric (see Section 2.4) are shown in Table 11. A couple of quick mesh adaptivity trials were conducted with different combinations of the interpolation error bound values to come up a good set of values as given in Table 11. Absolute interpolation error values were used here instead of the relative ones to ensure consistency in the specification of mesh resolution over space.

Table 11

Interpolation error bound for the fields that the mesh was adapted to.

\begin{tabular}{lc}
\hline Field & $\begin{array}{c}\text { Interpolation error bound } \\
\text { (absolute) }\end{array}$ \\
\hline Sauter mean diameter $\left(d_{32}\right)$ & $2.5 \times 10^{-7} \mathrm{~m}$ \\
Water velocity $\left(\mathbf{u}_{d}\right)$ & $(0.01,0.005) \mathrm{m} \mathrm{s}^{-1}$ \\
\hline
\end{tabular}

A number of other numerical parameters associated with the mesh adaptivity settings were adjusted and their values are shown in Table 12. Adaptivity subroutine was called after every 100 time steps, i.e. every $0.001 \mathrm{~s}$ in the simulation. This ensured that the mesh was adapted just enough so as to not change drastically between any two adapts, and the computational effort spent in adaptivity was also kept to a minimum. Therefore, a total of 1000 mesh adaptivity cycles were carried out in an overall simulation time of $1 \mathrm{~s}$. Bounds were specified for the minimum and maximum mesh edge lengths to prevent the mesh from becoming excessively fine or coarse. The consistent interpolation method was used to interpolate the various scalar, vector and tensor fields from the pre-adapt mesh to the post-adapt mesh. More details on the implementation of mesh adaptivity in Fluidity can be found in the Fluidity Manual (AMCG, 2015).

Since the simulation was performed in a parallelised framework 
on a multi-core machine, 15 adapt iterations had to be performed during each adaptivity cycle instead of just one. Nodes shared between different processors remain locked while the individual processors optimise their meshes, and load balancing is only performed after an adaptive iteration is completed. Hence, 15 adapt iterations were necessary to ensure that all nodes were considered for the adaptivity process at least once (Devine et al., 2002; AMCG, 2015).

\section{Table 12}

Adaptivity settings used for the adaptive mesh simulation of the polydispersed water-in-oil emulsion in Fluidity.

\begin{tabular}{lc}
\hline Number of time steps between two adapts & 100 \\
Gradation parameter & 1.3 \\
Minimum edge length (m) & $10^{-4}$ \\
Maximum edge length (m) & 0.01 \\
Number of adaptive iterations for parallel adaptivity & 15 \\
Field interpolation method & consistent \\
\hline
\end{tabular}

All flow and population balance discretisations were exactly the same as for the fixed mesh case in Section 4.2. The unconditionally-stable first-order upwind scheme was used to discretise the convective fluxes in the advection equations for volume fraction and population balance equation scalars. Other conditionally-stable schemes (such as finite element, trapezoidal, etc.) are stable for only a range of values of the grid Peclet number (Donea and Huerta, 2003), even when used in conjunction with slope limiters. Mesh adaptivity can lead to the generation of coarse elements which can then result in high values for the Peclet number in those regions making such schemes sensitive to the adapting mesh. The use of the unconditionallystable first-order upwind scheme in this work avoids this problem.

Initial mesh for the adaptive simulation was generated using the open-source mesh generation software Gmsh (Geuzaine and Remacle, 2009). Simulation results for the adaptive mesh simulation described above are shown in Figure 17. Water Sauter mean diameter and the corresponding adapted mesh are presented for three different simulation times. Although the mesh was also adapted to the two components of water velocity, water velocity contours have not been shown here. The mesh in Figure 17 clearly displays its non-homogeneous, anisotropic character, as expected, and conforms with the variations in the SMD over space. Elongated elements resolved the recirculation zone boundaries by concentrating node density in only the normal direction, by using the anisotropic property of the adapted mesh. Mesh anisotropy in the y-direction in the interior of the BFS comes from the inclusion of the flow velocity to the mesh adaptivity parameters.

A plot of the Sauter mean diameter along a vertical line $5 \mathrm{~mm}$ from the step is presented in Figure 18 for comparing the adaptive mesh simulation to two fixed meshes-Coarse mesh and Fine mesh. The Adaptive mesh was able to resolve the primary recirculation zone more accurately than the other two fixed meshes by increasing the mesh resolution near the recirculation zone boundary, and this can be seen from the steeper slope of the Sauter mean diameter plot between $y=4 \mathrm{~mm}$ and $y=6 \mathrm{~mm}$. Figure 18 also shows that the Adaptive mesh was able to resolve the top boundary layer better than the other two meshes. All this was made possible by optimising the placement of mesh nodes in the appropriate regions.

Figures 19 and 20 show the number of mesh nodes and runtimes, respectively for the Adaptive mesh simulation. The number of nodes for the Adaptive mesh simulation, as shown in Figure 19, always stayed below the number of Coarse mesh nodes with the maximum number of Adaptive mesh nodes still 30 percent lower than the fixed Coarse mesh. This number was much lower when compared to the Fine mesh. Similar behaviour was also displayed in the comparison of the simulation runtimes for the three meshes, as seen in Figure 20. The Adaptive mesh simulation took 30 percent less time to run than what the Coarse mesh took. Since this runtime for the Adaptive mesh simulation included both the solution computation time and the time needed for mesh adaptivity, it is clear that the cost of adaptivity was negligible compared to the solution computation time for this problem. The cost associated with an expensive interpolation method, such as the Galerkin projection interpolation, in the presence of large number of flow fields could potentially decrease the overall improvement in the efficiency obtained using mesh adaptivity, but it clearly was not the case in this work that used the consistent interpolation method.

It was shown in the previous paragraphs that the adaptive mesh simulation in this work was able to produce non-homogeneous, anisotropic, unstructured meshes, which conformed well with the variations in the water droplet size distribution. Moreover, the selected adaptive mesh simulation was quicker than both the fixed Coarse and Fine meshes, with the time taken for mesh adaptivity being small. Still, it needs to be shown quantitatively that the overall solution produced by the Adaptive mesh simulation was more accurate than the fixed mesh, if this technique is to serve any importance in the modelling of polydispersed multiphase flows. Figure 21 presents the $L^{2}$-norm of the error in the Sauter mean diameter (of water drops) with time, for the three meshes. The errors for the Adaptive mesh simulation were lower than those for the Coarse mesh beyond $t=0.4 \mathrm{~s}$. All errors here were calculated with respect to the Superfine fixed mesh solution, as discussed in Section 4.2. The Adaptive mesh solution error was 40 percent smaller than the Coarse mesh error at $t=1 \mathrm{~s}$.

It can be summarised that mesh adaptivity produced quicker and more accurate solution than the corresponding fixed mesh simulation in this work. It is safe to generalise here that polydispersed multiphase flows can be simulated more efficiently using this technique when appropriate mesh adaptivity parameters are chosen in the simulation. The cost of mesh adaptivity in these simulations is negligible when compared to the solution computation time and hence should be preferred over other fixed solvers when simulating polydispersed multiphase flows in two or three spatial dimensions. 

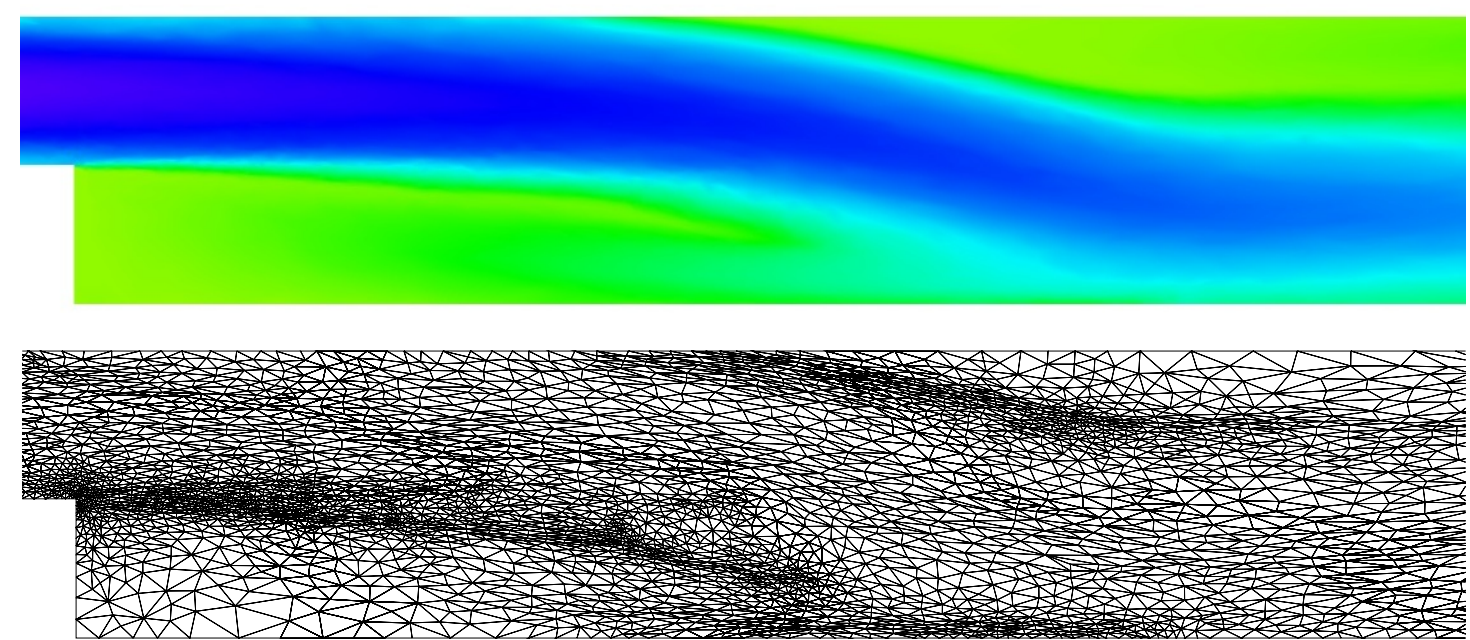

(a) $t=0.50 \mathrm{~s}$
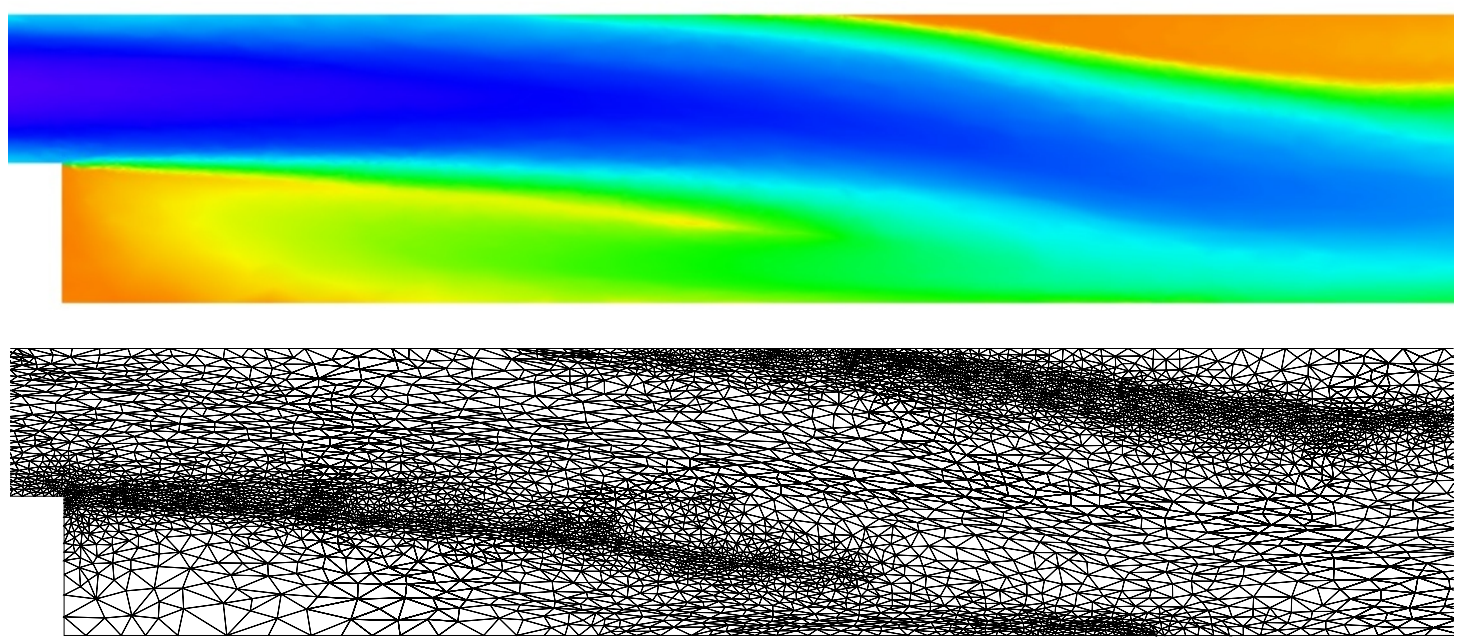

(b) $t=0.75 \mathrm{~s}$
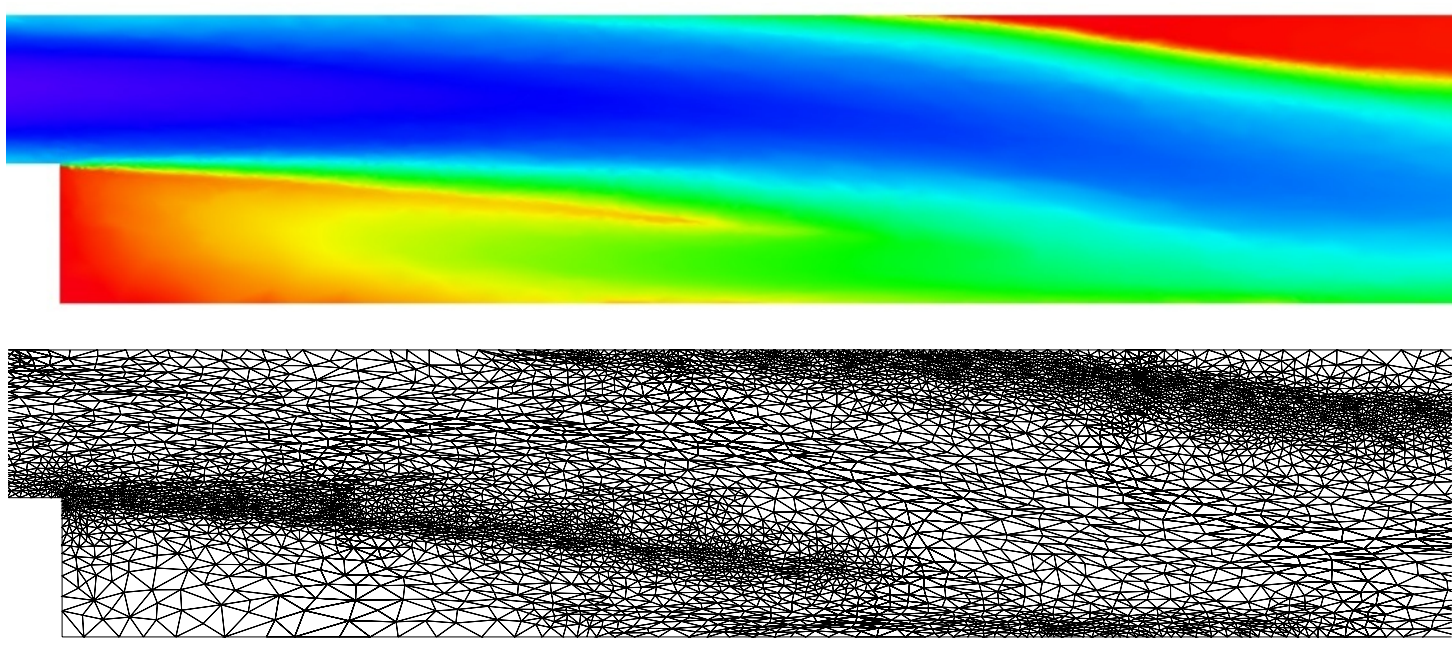

(c) $t=1.0 \mathrm{~s}$

Water Sauter mean diameter $(\mu \mathrm{m})$

\begin{tabular}{lllll}
\hline 50 & 58 & 66 & 74 & 82
\end{tabular}

Figure 17: Water Sauter mean diameter and the corresponding mesh for the adaptive mesh simulation of the polydispersed water-in-oil emulsion. (a) $t=0.50 \mathrm{~s}$, (b) $\mathrm{t}=0.75 \mathrm{~s}$ and $(\mathrm{c}) \mathrm{t}=1.0 \mathrm{~s}$. 


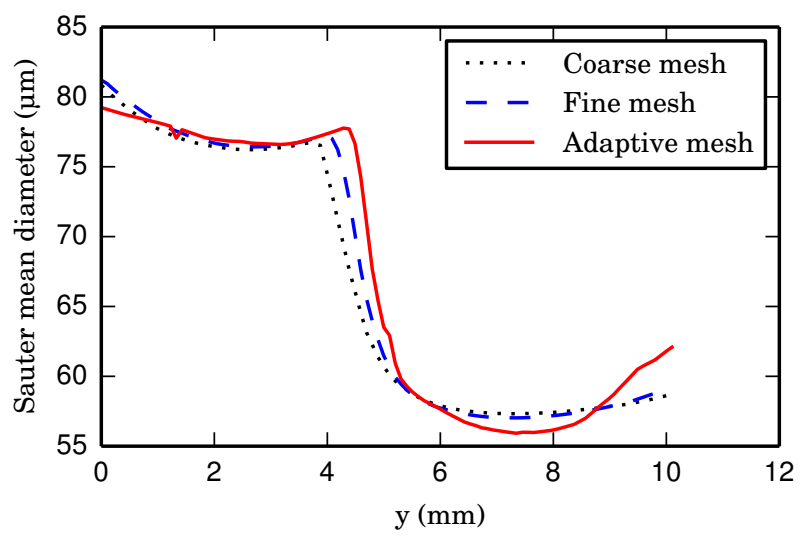

Figure 18: Sauter mean diameter of the water drops plotted along a vertical line $5 \mathrm{~mm}$ from the step of the BFS. Result for the Adaptive mesh is compared with the Coarse mesh and the Fixed mesh results.

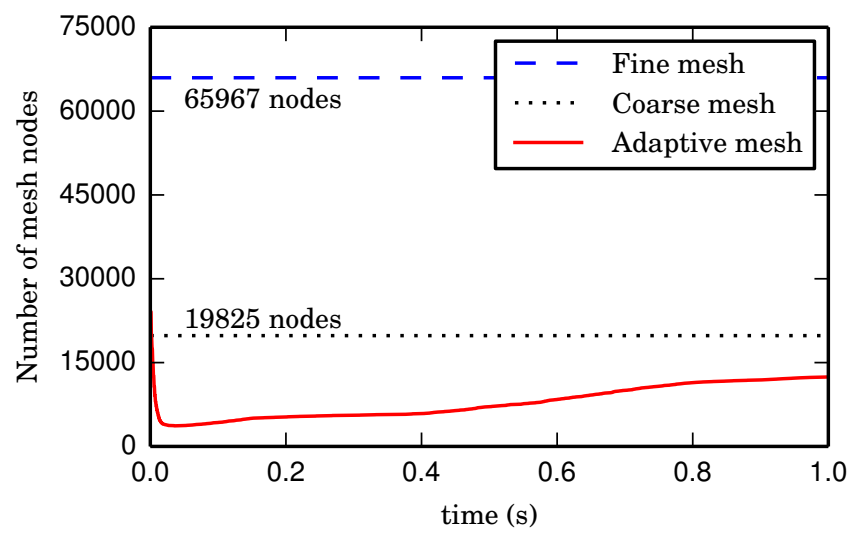

Figure 19: Plot of the number of mesh nodes with time for the Adaptive mesh, Coarse mesh and the Fine mesh.

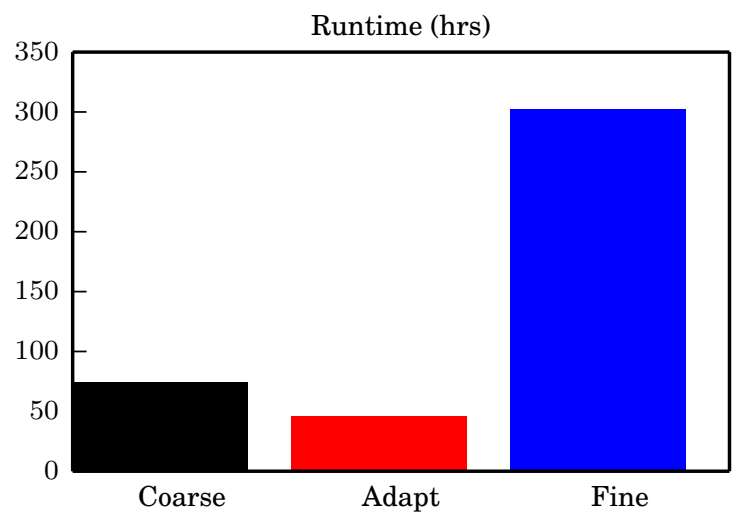

Figure 20: Runtimes (walltime) compared for the Coarse mesh, Adaptive mesh and Fine mesh simulations. All runtimes are presented for 8-core parallel runs. Mesh was adapted after every $0.001 \mathrm{~s}$, i.e. a total of 1000 mesh adaptivity cycles in the $1 \mathrm{~s}$ simulation.

\section{Conclusions}

An efficient, open-source finite element framework to solve polydispersed flow problems was presented in this paper. CFD based modelling of polydispersed flows in the open-source Flu-

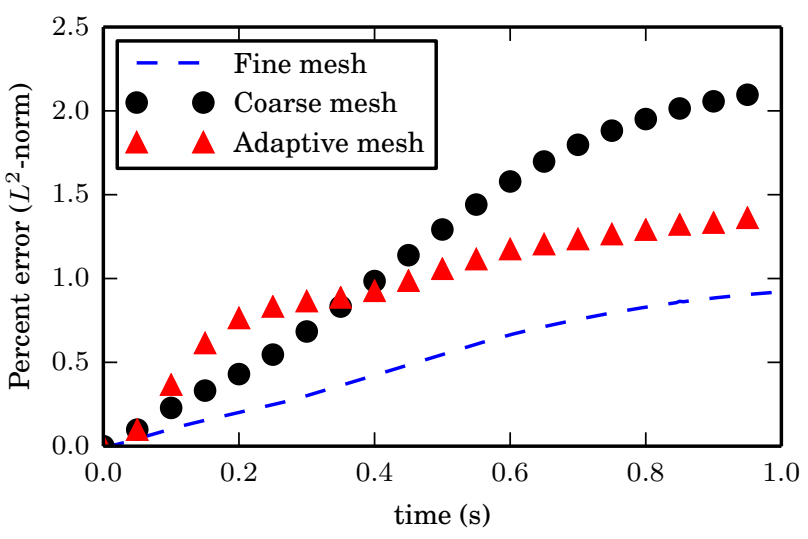

Figure 21: Error in the Adaptive mesh simulation compared with Coarse and Fine mesh results. $L^{2}$-norm of the percentage error in the Sauter mean diameter has been plotted here with time.

idity code was implemented using a coupled Eulerian-Eulerian population balance modelling approach. Marchisio and Fox (2005)'s direct quadrature method of moments was utilised for solving the population balance equation and the implementation was verified successfully using McCoy and Madras (2003)'s analytical solution.

The present implementation is general for up to three spatial dimensions, but for simplicity the code was benchmarked against a commercial CFD software for a hypothetical two-dimensional water-in-oil emulsion problem in a backward facing step domain. A hybrid finite element-control volume method for solving the coupled system of equations gave the best result. In this method the velocity-pressure equation set was discretised using the $\mathrm{P} 1_{\mathrm{DG}}-\mathrm{P} 2$ element pair and the volume fraction equation and the population balance equation were discretised using the control volume approach. A good match between the results produced by the present implementation and the commercial CFD software ANSYS Fluent, was obtained. Although a few other discretisations were tested for the population balance equation, the hybrid FE-CV method was the most robust of all as the $\mathrm{CV}$ method was conservative and stable.

The hypothetical backward facing step problem in this work was explicitly assumed to be laminar to demonstrate the coupling between the Eulerian-Eulerian flow equations with the population balance equation without bringing in the complexities of modelling turbulence. Real applications, though, are seldom laminar and turbulence equations need to be coupled to the above set of equations, which will be discussed in future work by the authors.

Adaptive mesh refinement was also applied to the polydispersed BFS simulation. The finite element mesh in this case was adapted in time to the water Sauter mean diameter and velocity fields so that these fields satisfied a specific resolution requirement on the resulting adapted mesh. Fully-unstructured, nonhomogeneous, anisotropic meshes were generated by the mesh adaptivity subroutine. The adapted meshes conformed remarkably with the flow fields that they were adapted to. An excellent improvement in the solver efficiency was reported by the 
use of mesh adaptivity in this problem. The errors produced by the adaptive mesh simulation were 40 percent smaller than the corresponding errors in the fixed mesh simulation. The adaptive mesh simulation, after taking into account the time taken to adapt meshes, was 35 percent faster than the corresponding fixed mesh simulation.

Based on the results obtained in this work, it can be concluded that the open-source finite element framework of Fluidity is accurate and provides an efficient alternative to the other available finite volume/ control volume CFD packages for solving polydispersed flow problems. The present implementation allows for a single continuous phase with many dispersed phases, with each dispersed phase containing as many population balance equations as required. Experimental validation and the use of more than one population balance equation per phase to model a three-phase three-dimensional industrial polydispersed flow problem will be presented in the future work by the authors. Although the current implementation can handle only one internal variable in the NDF, the ability to calculate multidimensional moments of the NDF will be added in the future to model more complex dispersed phases. With the use of mesh adaptivity in a highly-parallelised distributed computational environment, the open-source finite element framework presented in this paper (employing the population balance modelling approach) can prove to be an efficient tool for modelling industrial-scale multiphase polydispersed flow problems.

\section{Acknowledgements}

We would like to thank the members of the Applied Modelling and Computation Group with whom we had interesting discussions regarding the work presented in this paper. We also acknowledge the support received from the High Performance Computing Service at Imperial College London. Special thanks to Sam Parkinson for the initial work on the implementation of DQMOM in Fluidity. GB would like to thank Ajayendar Reddy and Deepak Sachan for proofreading a first draft of this manuscript and providing their valuable suggestions.

\section{Appendix A. Length-based formulation of the analytical solution}

The length-based version of the problem developed by McCoy and Madras (2003) can be written as follows:

For an initial number density function given by

$$
n_{\xi}(\xi, 0)=3 \xi^{2} \frac{m_{0}(0)^{2}}{m_{3}} \exp \left(-\xi^{3} \frac{m_{0}(0)}{m_{3}}\right),
$$

and breakage and aggregation kernels given by

$$
\begin{gathered}
v=2, \\
a(\xi)=\tilde{s} \xi^{3},
\end{gathered}
$$

$$
b\left(\xi \mid \xi_{1}\right)= \begin{cases}\frac{3 \xi^{2}}{\xi_{1}^{3}} & \text { if } \xi<\xi_{1} \\ 0 & \text { otherwise }\end{cases}
$$

and

$$
\beta=\text { constant, }
$$

there exists an analytical solution to the population balance equation (Equation (9)) when $\mathbf{u}=0$, which is given as:

$$
n_{\xi}(\xi, t)=k_{1} \xi^{2} \exp \left(-k_{2} \xi^{3}\right) .
$$

$k_{1}$ and $k_{2}$ here are given by:

$$
k_{1}=3 \hat{n}_{\xi}^{2}(t) \frac{m_{0}(0)^{2}}{m_{3}}
$$

and

$$
k_{2}=\hat{n}_{\xi}(t) \frac{m_{0}(0)}{m_{3}}
$$

where

$$
\hat{n}_{\xi}(t)=\hat{n}_{\xi}(\infty)\left[\frac{1+\hat{n}_{\xi}(\infty) \tanh \left(\hat{n}_{\xi}(\infty) \frac{m_{0}(0) \beta t}{2}\right)}{\hat{n}_{\xi}(\infty)+\tanh \left(\hat{n}_{\xi}(\infty) \frac{m_{0}(0) \beta t}{2}\right)}\right] .
$$

The constant $\hat{n}_{\xi}(\infty)$ is given in terms of the breakage and aggregation kernels as:

$$
\hat{n}_{\xi}(\infty)=\left(\frac{2 \tilde{s} m_{3}}{\beta}\right)^{1 / 2}\left(\frac{1}{m_{0}(0)}\right) .
$$

$m_{0}(0)$ and $m_{3}$ in all the above equations are specified as initial conditions.

Since DQMOM was used in this work, the moments of the NDF given in Equation (A.6) were needed for the calculation of the Sauter mean diameter, $d_{32}$, for verification. The first four length-based moments of this function can be written as:

$$
\begin{aligned}
& m_{0}(t)=m_{0}(0) \hat{n}_{\xi}(t), \\
& m_{1}(t)=\frac{k_{1}}{3 k_{2}{ }^{4 / 3}} \Gamma\left(\frac{4}{3}\right), \\
& m_{2}(t)=\frac{k_{1}}{3 k_{2}{ }^{5 / 3}} \Gamma\left(\frac{5}{3}\right),
\end{aligned}
$$

and

$$
m_{3}(t)=m_{3},
$$

where $\Gamma$ is the standard gamma function. These equations were also used for the calculation of the initial moment set for initialising the polydispersed flow problem.

\section{References}

Abbasi E, Arastoopour H. Numerical analysis and implementation of finite domain complete trial functions method of moments (FCMOM) in CFD codes. Chem Eng Sci 2013;102:432-441.

AMCG. Fluidity manual v4.1.12. Imperial College London 2015 04;http://dx.doi.org/10.6084/m9.figshare.1387713. 


\section{Nomenclature}

\section{Abbreviations}

BFS

$\mathrm{CG}$

$\mathrm{CV}$

DG

DQMOM

FE

FEM

NDF

PBE

QMOM

SMD

SUPG

\section{Uppercase Roman Symbols}

$B_{B} \quad$ Birth term due to breakage

$B_{C} \quad$ Birth term due to coalescence

$C_{D} \quad$ Drag coefficient used in the interphase drag force term

$D_{B} \quad$ Death term due to breakage

$D_{C} \quad$ Death term due to coalescence

$H \quad$ Hessian tensor of the solution field (in mesh adaptivity)

$M \quad$ Riemannian mesh metric used in mesh adaptivity

$N \quad$ Number of weights or abscissas used in the approximation of the number density function in the DQMOM method

$N_{\text {phases }} \quad$ Number of phases in the multiphase problem

$R e_{c} \quad$ Continuous phase Reynolds number

$R e_{d} \quad$ Dispersed phase Reynolds number

$S_{\xi} \quad$ Source term in the population balance equation

Lowercase Roman Symbols

$\tilde{s} \quad$ Constant coefficient in the power law breakage frequency

a Breakage frequency

$b$ Breakage daughter distribution function

$d \quad$ Dispersed phase diameter

$d_{32} \quad$ Sauter mean diameter

\section{Greek Symbols}

$\alpha$

$\beta$

$\epsilon$

$\mu$

$v$

$\rho$

$\checkmark$

$\xi$

$\langle\xi\rangle_{j}$

$\overline{\bar{\tau}}_{i}$

\section{Subscripts}

$c, d$ $j^{\text {th }}$ source term in the DQMOM weight equation

$j^{\text {th }}$ source term in the DQMOM weighted abscissa equation

$k^{\text {th }}$ moment of the number density function

Number density function used in the PBE

Common pressure field for all phases

Time coordinate

$\mathrm{X}$-component of velocity

$j^{\text {th }}$ weight in the DQMOM approximation of the NDF

Interphase drag force

Interphase force acting on phase $i$ due to all other phases

Acceleration due to gravity

Velocity of phase $i$

Vector of external coordinates in the NDF, or spatial coordinates

$\mathrm{y}$-component of velocity

Volume fraction

Aggregation frequency

User-specified interpolation error bound for mesh adaptivity

Dynamic viscosity

Average number of dispersed particles formed after breakage

Density

Weighted abscissa in the DQMOM equation $\left(\varsigma_{j}=\right.$ $\left.w_{j}\langle\xi\rangle_{j}\right)$

Internal coordinate in the NDF

$j^{\text {th }}$ abscissa in the DQMOM approximation of the NDF

Deviatoric stress tensor for phase $i$

Continuous and dispersed phases, respectively

Phase 
Armaly BF, Durst F, Pereira J, Schönung B. Experimental and theoretical investigation of backward-facing step flow. J Fluid Mech 1983;127:473-496.

Babuška I, Rheinboldt WC. Error estimates for adaptive finite element computations. SIAM J Numer Anal 1978;15(4):736-754.

Bannari R, Kerdouss F, Selma B, Bannari A, Proulx P. Three-dimensional mathematical modeling of dispersed two-phase flow using class method of population balance in bubble columns. Comput Chem Eng 2008;32(12):3224 3237.

Bassi F, Rebay S. A high-order accurate discontinuous finite element method for the numerical solution of the compressible Navier-Stokes equations. J Comput Phys 1997;131(2):267-279.

Becker PJ, Puel F, Dubbelboer A, Janssen J, Sheibat-Othman N. Coupled population balance-CFD simulation of droplet breakup in a high pressure homogenizer. Comput Chem Eng 2014;68:140-150.

Bowen RM. Theory of mixtures. In: Eringen AC, editor. Continuum physics, vol. III Waltham: Academic Press; 1976.

Brennen CE. Fundamentals of multiphase flow. Cambridge University Press; 2005.

Buffo A, Vanni M, Marchisio DL. Multidimensional population balance model for the simulation of turbulent gas-liquid systems in stirred tank reactors. Chem Eng Sci 2012;70:31-44.

Buwa VV, Ranade VV. Dynamics of gas-liquid flow in a rectangular bubble column: experiments and single/multi-group CFD simulations. Chem Eng Sci 2002;57(22):4715-4736.

Chan T, Liu Y, Chan C. Direct quadrature method of moments for the exhaust particle formation and evolution in the wake of the studied ground vehicle. J Aerosol Sci 2010;41(6):553-568.

Chen P, Sanyal J, Dudukovic M. CFD modeling of bubble columns flows: implementation of population balance. Chem Eng Sci 2004;59(22):52015207.

Clift R, Grace JR, Weber ME. Bubbles, drops, and particles. Academic Press; 1978.

Cotter CJ, Ham DA, Pain CC. A mixed discontinuous/continuous finite element pair for shallow-water ocean modelling. Ocean Modell 2009;26(1):86-90.

Devine K, Boman E, Heaphy R, Hendrickson B, Vaughan C. Zoltan data management services for parallel dynamic applications. Comput Sci Eng 2002;4(2):90-96.

Donea J, Huerta A. Finite element methods for flow problems. John Wiley \& Sons; 2003.

Elman HC, Silvester DJ, Wathen AJ. Finite elements and fast iterative solvers: with applications in incompressible fluid dynamics. Oxford University Press; 2014

Farrell P, Piggott M, Pain C, Gorman G, Wilson C. Conservative interpolation between unstructured meshes via supermesh construction. Comput Meth Appl Mech Eng 2009;198(33):2632-2642.

Favero JL, Silva LFLR, Lage PLC. Modeling and simulation of mixing in water-in-oil emulsion flow through a valve-like element using a population balance model. Comput Chem Eng 2015;75:155-170.

Frenklach M, Harris SJ. Aerosol dynamics modeling using the method of moments. J Colloid Interface Sci 1987;118(1):252-261.

Geuzaine C, Remacle JF. Gmsh: A 3-D finite element mesh generator with built-in pre-and post-processing facilities. Int J Numer Meth Eng 2009;79(11):1309-1331.

Gordon RG. Error bounds in equilibrium statistical mechanics. J Math Phys 1968;9:655.

Gresho PM, Chan ST. Solving the incompressible Navier-Stokes equations using consistent mass and a pressure Poisson equation. Lawrence Livermore National Lab., CA (USA); 1988.

Ishii M, Zuber N. Drag coefficient and relative velocity in bubbly, droplet or particulate flows. AlChE J 1979;25(5):843-855.

Jacobs CT, Collins GS, Piggott MD, Kramer SC, Wilson CRG. Multiphase flow modelling of volcanic ash particle settling in water using adaptive unstructured meshes. Geophys J Int 2013;192(2):647-665.

Marchal P, David R, Klein J, Villermaux J. Crystallization and precipitation engineering-I. An efficient method for solving population balance in crystallization with agglomeration. Chem Eng Sci 1988;43(1):59-67.

Marchisio DL, Fox RO. Computational models for polydisperse particulate and multiphase systems. Cambridge University Press; 2013.

Marchisio DL, Fox RO. Solution of population balance equations using the direct quadrature method of moments. J Aerosol Sci 2005;36(1):43-73.

Marchisio DL, Pikturna JT, Fox RO, Vigil RD, Barresi AA. Quadrature method of moments for population-balance equations. AlChE J 2004;49(5):12661276.

McClure DD, Norris H, Kavanagh JM, Fletcher DF, Barton GW. Validation of a computationally efficient computational fluid dynamics (CFD) model for industrial bubble column bioreactors. Ind Eng Chem Res 2014;53(37):1452614543

McCoy B, Madras G. Analytical solution for a population balance equation with aggregation and fragmentation. Chem Eng Sci 2003;58(13):30493051.

McGraw R. Description of aerosol dynamics by the quadrature method of moments. Aerosol Sci Technol 1997;27(2):255-265.

Pain C, Umpleby A, De Oliveira C, Goddard A. Tetrahedral mesh optimisation and adaptivity for steady-state and transient finite element calculations. Comput Meth Appl Mech Eng 2001;190(29):3771-3796.

Piggott M, Farrell P, Wilson C, Gorman G, Pain C. Anisotropic mesh adaptivity for multi-scale ocean modelling. Philos Trans R Soc London, Ser A 2009;367(1907):4591-4611.

Prakash AV, Chaudhury A, Barrasso D, Ramachandran R. Simulation of population balance model-based particulate processes via parallel and distributed computing. Chem Eng Res Des 2013;91(7):1259-1271.

Prosperetti A, Tryggvason G. Computational methods for multiphase flow. Cambridge university press; 2007.

Ramkrishna D. Population balances: Theory and applications to particulate systems in engineering. Academic press; 2000.

Roudsari SF, Turcotte G, Dhib R, Ein-Mozaffari F. CFD modeling of the mixing of water in oil emulsions. Comput Chem Eng 2012;45:124-136.

Santos FP, Senocak I, Favero JL, Lage PLC. Solution of the population balance equation using parallel adaptive cubature on GPUs. Comput Chem Eng 2013;55:61-70

Sanyal J, Marchisio DL, Fox RO, Dhanasekharan K. On the comparison between population balance models for CFD simulation of bubble columns. Ind Eng Chem Res 2005;44(14):5063-5072.

Schiller L, Naumann Z. A drag coefficient correlation. Vdi Zeitung 1935;77(318):51.

Selma B, Bannari R, Proulx P. Simulation of bubbly flows: Comparison between direct quadrature method of moments (DQMOM) and method of classes (CM). Chem Eng Sci 2010;65(6):1925-1941.

Silva L, Damian R, Lage P. Implementation and analysis of numerical solution of the population balance equation in CFD packages. Comput Chem Eng 2008;32(12):2933-2945.

Silva L, Lage P. Development and implementation of a polydispersed multiphase flow model in OpenFOAM. Comput Chem Eng 2011;35(12):26532666

Sweby PK. High resolution schemes using flux limiters for hyperbolic conservation laws. SIAM J Numer Anal 1984;21(5):995-1011.

Szilágyi B, Agachi Pc, Barabás R, Lakatos BG. Coupled Population BalanceCFD Modelling of a Continuous Precipitation Reactor. In: Klemeš JJ, Varbanov PP, Liew PY, editors. 24th European Symposium on Computer Aided Process Engineering, vol. 33 of Computer Aided Chemical Engineering; 2014.p. 187-192.

Tabib MV, Roy SA, Joshi JB. CFD simulation of bubble column-an analysis of interphase forces and turbulence models. Chem Eng J 2008;139(3):589_ 614.

Vasilevskii YV, Lipnikov K. An adaptive algorithm for quasioptimal mesh generation. Comput Math Math Phys 1999;39(9):1468-1486.

Wilson C. Modelling multiple-material flows on adaptive unstructured meshes. $\mathrm{PhD}$ thesis, Imperial College London; 2009.

Yeoh G, Cheung S, Tu J. On the prediction of bubble size distribution and void fraction in vertical gas-liquid flows. J Comput Multiphase Flows 2012;4(1):1-22.

Yeoh GH, Cheung CP, Tu J. Multiphase flow analysis using population balance modeling: bubbles, drops and particles. Butterworth-Heinemann; 2013.

Zucca A, Marchisio DL, Barresi AA, Fox RO. Implementation of the population balance equation in CFD codes for modelling soot formation in turbulent flames. Chem Eng Sci 2006;61(1):87-95. 